\title{
Matroids on Convex Geometries: Subclasses, Operations, and Optimization
}

\author{
by
}

Yoshio SANO

\begin{abstract}
A matroid-like structure defined on a convex geometry, called a cg-matroid, was introduced by S. Fujishige, G. A. Koshevoy, and Y. Sano [Matroids on convex geometries (cg-matroids), Discrete Math. 307 (2007) 1936-1950]. In this paper, we continue the study of cg-matroids and extend the theory of cg-matroids. We give some characterizations of cg-matroids by axioms. Strict cg-matroids are a special subclass of cg-matroids which have nice properties. We define another subclass of cg-matroids, called co-strict cg-matroids, which also have good properties. Moreover, we consider operations on cg-matroids such as restriction and contraction. These operations are closely related to subclasses of cg-matroids. We also consider an optimization problem on cg-matroids, which reveals the relation between the greedy algorithm and cg-matroids.
\end{abstract}

2010 Mathematics Subject Classification: 05B35, 52B40, 90C27.

Keywords: matroid, convex geometry, independent set, spanning set.

\section{Contents}

1 Introduction 672

2 Preliminaries on convex geometries 674

2.1 Notation 674

2.2 Convex geometries 674

2.3 Operations on convex geometries 676

3 Matroids on convex geometries (cg-matroids) 679

3.1 Definition 679

3.2 Combinatorial structure of cg-matroids 680

4 Subclasses of cg-matroids 684

4.1 Strict cg-matroids 684

4.2 Co-strict cg-matroids 686

4.3 Tame and wild cg-matroids 689

Communicated by S. Iwata. Received July 15, 2009. Revised November 17, 2009.

Y. Sano: RIMS, Kyoto University, Kyoto 606-8502, Japan;

e-mail: sano@kurims.kyoto-u.ac.jp

(C) 2011 Research Institute for Mathematical Sciences, Kyoto University. All rights reserved. 
5 Operations on cg-matroids 691

5.1 Restriction 691

5.2 Contraction 693

5.3 Other operations 695

6 Optimization on cg-matroids 697

6.1 Maximum base problem and the greedy algorithm 697

6.2 Characterization 698

References 702

\section{$\S 1$. Introduction}

The notion of a matroid was introduced by H. Whitney [17] in 1935 as an abstraction of the notion of linear independence in a vector space. The importance of matroids for combinatorial optimization was revealed in the 1960 s by J. Edmonds, who found efficient algorithms and min-max relations for optimization problems involving matroids (see [6]). Matroids are exactly those structures where the greedy algorithm yields an optimum solution. Many researchers have studied and extended the matroid theory (see books by D. J. A. Welsh [16], J. Oxley [11], A. Schrijver [14] and S. Fujishige [8]).

The notion of a poset matroid which was studied by M. Barnabei, G. Nicoletti, and L. Pezzoli [2], [3] in the 1990s as a generalization of a matroid. It is a matroidlike structure defined on a partially ordered set (poset) instead of just a finite set. Poset matroids are equivalent to supermatroids defined on distributive lattices; the notion of a supermatroid was introduced by F. D. J. Dunstan, A. W. Ingleton, and D. J. A. Welsh [4] in 1972 as a generalization of the concept of a matroid (see also [12], [15] for related topics).

A matroid-like structure defined on a convex geometry, called a cg-matroid, was introduced by S. Fujishige, G. A. Koshevoy, and Y. Sano [9] in 2007 as a generalization of a (poset) matroid. A convex geometry on a nonempty finite set $E$ is the pair of the set $E$ and an intersection-closed family $\mathcal{F}$ of subsets of $E$, called the family of closed sets, which contains the empty set and the set $E$ and satisfies the "one-point extension property". The notion of a convex geometry arose from the notion of convexity in a vector space and was introduced by P. H. Edelman and R. E. Jamison [5]. Note that a convex geometry is the dual of an antimatroid. A cg-matroid is defined to be the pair of a convex geometry and a nonempty subfamily $\mathcal{B}$ of the family of closed sets of the convex geometry, called the family of bases, which is a clutter satisfying a "middle base property". Fujishige et al. showed that all the bases of a cg-matroid have the same cardinality, and gave a characterization of a cg-matroid by an "exchange property" for bases. Here appears one of the significant differences between cg-matroids and ordinary matroids: In 
an ordinary matroid, if we have two bases $B_{1}, B_{2}$ and an element $e_{1}$ in the base $B_{1}$ then we can find an element $e_{2}$ in the other base $B_{2}$ so that the base $B_{1}$ with $e_{1}$ replaced by $e_{2}$ is also a base of the matroid. In a cg-matroid, there is no guarantee that we can find such an element $e_{2}$ in the base $B_{2}$ but still we can find such an element $e_{2}$ in the closure of the union of the bases $B_{1}$ and $B_{2}$. An independent set of a cg-matroid is a closed subset of a base of the cg-matroid. Fujishige et al. also gave a characterization of cg-matroids by axioms for independent sets.

In this paper, we continue the study of cg-matroids and extend their theory. This paper is organized as follows: In Section 2, we prepare some notation which will be used in this paper and recall the definition and some properties of convex geometries. In Section 3, we first recall the definition and basic results for cgmatroids, which were given in [9]. Then we give another characterization of cgmatroids. A spanning set of a cg-matroid is a closed set which contains a base of the cg-matroid. We characterize cg-matroids by axioms for spanning sets.

In Section 4, we consider some subclasses of cg-matroids which arise naturally from the characterization of cg-matroids obtained in Section 3 by strengthening an axiom in the characterizations. A cg-matroid satisfying the strict augmentation property is said to be strict, and a cg-matroid satisfying the strict reduction property is said to be co-strict. Strict cg-matroids are a special subclass of cg-matroids on which we can define the rank functions naturally. Characterizations of strict cg-matroids by axioms for rank functions were given in [13]. In Section 4, we give some characterizations of strict cg-matroids and co-strict cg-matroids.

In Section 5, we consider operations on cg-matroids such as restriction and contraction. These operations are closely related to the subclasses of cg-matroids given in Section 4. The restriction of a cg-matroid to a spanning set is also a cgmatroid, but the restriction of a cg-matroid to a closed set is not a cg-matroid in general. In the case of a strict cg-matroid, the restriction to a closed set is always a strict cg-matroid. Similarly, the contraction of a cg-matroid by an independent set is also a cg-matroid, but the contraction of a cg-matroid by a closed set is not a cg-matroid in general. In the case of a co-strict cg-matroid, the contraction by a closed set is always a co-strict cg-matroid.

In Section 6, we consider an optimization problem on cg-matroids, which reveals the relation between the greedy algorithm and cg-matroids. For a given hereditary system on a convex geometry and a nonnegative weight function on the ground set, we consider the maximum base problem, or the maximum independent set problem. We show that if the hereditary system is a strict cg-matroid and the weight function is "natural" on the convex geometry, then the greedy algorithm always produces an optimal solution of the maximum independent set problem. We 
also show that a hereditary system on a convex geometry with the property that the greedy algorithm produces an optimal solution of the problem for any natural nonnegative weight function on the convex geometry is a strict cg-matroid. This gives a characterization of strict cg-matroids by the greedy algorithm.

\section{$\S 2$. Preliminaries on convex geometries}

\section{$\S 2.1$. Notation}

In this subsection, we prepare some notation which is used in this paper. Let $E$ be a nonempty finite set. We denote the family of all subsets of $E$ by $2^{E}$. For two sets $A$ and $B$, we denote the set $\{e \in A \mid e \notin B\}$ by $A \backslash B$. For a family $\mathcal{A}$ of subsets of $E$, we denote the set of all maximal elements (with respect to set inclusion) in the family $\mathcal{A}$ by $\operatorname{Max}(\mathcal{A})$, the set of all minimal elements in $\mathcal{A}$ by $\operatorname{Min}(\mathcal{A})$, the lower set of $\mathcal{A}$ in the set $2^{E}$ (endowed with a partial order $\subseteq$ ) by $\operatorname{Low}(\mathcal{A}$ ), and the upper set of $\mathcal{A}$ in $2^{E}$ by $\operatorname{Upp}(\mathcal{A})$ :

$$
\begin{aligned}
& \operatorname{Low}(\mathcal{A}):=\left\{X \in 2^{E} \mid \exists A \in \mathcal{A}: X \subseteq A\right\}, \\
& \operatorname{Upp}(\mathcal{A}):=\left\{X \in 2^{E} \mid \exists A \in \mathcal{A}: X \supseteq A\right\} .
\end{aligned}
$$

Definition. For a family $\mathcal{A}$ of subsets of $E$ and a subset $X$ of $E$, we define the following families:

$$
\begin{aligned}
& \mathcal{A}^{(X)}:=\operatorname{Low}(\{X\}) \cap \mathcal{A}=\{A \mid A \in \mathcal{A}, A \subseteq X\} \subseteq 2^{X} \subseteq 2^{E}, \\
& \mathcal{A}_{[X]}:=\operatorname{Upp}(\{X\}) \cap \mathcal{A}=\{A \mid A \in \mathcal{A}, A \supseteq X\} \subseteq 2^{E}, \\
& \mathcal{A}_{(X)}:=\left\{A \backslash X \mid A \in \mathcal{A}_{[X]}\right\} \subseteq 2^{E \backslash X} .
\end{aligned}
$$

We call $\mathcal{A}^{(X)}$ the (lower) restriction of $\mathcal{A}$ to $X, \mathcal{A}_{[X]}$ the upper restriction of $\mathcal{A}$ to $X$, and $\mathcal{A}_{(X)}$ the contraction of $\mathcal{A}$ by $X$.

\section{$\S 2.2$. Convex geometries}

A convex geometry is a fundamental combinatorial structure defined on a finite set (see P. H. Edelman and R. E. Jamison [5]).

Definition. Let $E$ be a nonempty finite set and $\mathcal{F}$ a family of subsets of $E$. The pair $(E, \mathcal{F})$ is called a convex geometry on $E$ if $\mathcal{F}$ satisfies the following three properties:

(CG0) $\emptyset, E \in \mathcal{F}$.

(CG1) $X, Y \in \mathcal{F} \Rightarrow X \cap Y \in \mathcal{F}$.

(CG2) For any $X \in \mathcal{F} \backslash\{E\}$, there exists $e \in E \backslash X$ such that $X \cup\{e\} \in \mathcal{F}$. 
The set $E$ is called the ground set of the convex geometry $(E, \mathcal{F})$, and each element of $\mathcal{F}$ is called a closed set. It should be noted that the property (CG2) is equivalent to the following property whenever the properties (CG0) and (CG1) hold (cf. [5, Theorem 2.2]):

$(\mathrm{CG} 2)^{\prime}$ Every maximal chain $\emptyset=X_{0} \subsetneq X_{1} \subsetneq \cdots \subsetneq X_{n}=E$ of closed sets of $(E, \mathcal{F})$ has length $n=|E|$.

Example 2.1. The following are examples of convex geometries.

(a) Let $E$ be a finite set of points in a Euclidean space $\mathbb{R}^{d}$. Define $\mathcal{F}=\left\{X \in 2^{E} \mid\right.$ $X=\operatorname{Conv}(X) \cap E\}$, where $\operatorname{Conv}(X)$ denotes the convex hull of $X$ in $\mathbb{R}^{d}$. Then $(E, \mathcal{F})$ is a convex geometry, called a convex shelling or an affine convex geometry.

(b) Let $E$ be the vertex set of a tree $T$. Define $\mathcal{F}=\left\{X \in 2^{E} \mid X\right.$ is the vertex set of a subtree of $T\}$. Then $(E, \mathcal{F})$ is a convex geometry, called a tree shelling.

(c) Let $E$ be a partially ordered set (poset). Define $\mathcal{F}=\left\{X \in 2^{E} \mid X\right.$ is an (order) ideal of $E$. Then $(E, \mathcal{F})$ is a convex geometry, called a poset shelling. It is well-known that a convex geometry $(E, \mathcal{F})$ is a poset shelling if and only if $\mathcal{F}$ is closed with respect to set union.

Next, we define operators associated with a convex geometry.

Definition. Let $(E, \mathcal{F})$ be a convex geometry. The closure operator of $(E, \mathcal{F})$ is an operator $\tau: 2^{E} \rightarrow \mathcal{F}$ defined by

$$
\tau(X)=\bigcap\{Y \mid Y \in \mathcal{F}, X \subseteq Y\} \quad\left(X \in 2^{E}\right) .
$$

That is, $\tau(X)$ is the unique minimal closed set containing $X$. The set $\tau(X)$ is called the closure of $X$.

The closure operator $\tau$ satisfies the following properties:

(c10) $\tau(\emptyset)=\emptyset$.

(cl1) $X \subseteq \tau(X)$ for any $X \in 2^{E}$ (Extensionality).

(cl2) $X \subseteq Y \Rightarrow \tau(X) \subseteq \tau(Y)$ for any $X, Y \in 2^{E}$ (Monotonicity).

(cl3) $\tau(\tau(X))=\tau(X)$ for any $X \in 2^{E}$ (Idempotence).

Note. In general, any operator $\tau: 2^{E} \rightarrow 2^{E}$ satisfying the four conditions given above is called a closure operator. Conversely, given a closure operator $\tau$, define $\mathcal{F}=\left\{X \in 2^{E} \mid \tau(X)=X\right\}$. Then $\mathcal{F}$ satisfies the properties (CG0) and (CG1). The pair $(E, \tau)$ of a set $E$ and a closure operator $\tau: 2^{E} \rightarrow 2^{E}$ is called a closure 
space. In terms of a closure operator, a closure space $(E, \tau)$ is a convex geometry if and only if it satisfies the following property:

(AE) (Anti-Exchange Property) $X \in 2^{E}, \quad p \in E \backslash \tau(X), q \in \tau(X \cup\{p\}) \backslash\{p\} \Rightarrow$ $p \notin \tau(X \cup\{q\})$.

It is well-known that a convex geometry forms a graded lattice with respect to set inclusion, where the lattice operations of join $\vee$ and meet $\wedge$ are given by $X \vee Y:=\tau(X \cup Y)$ and $X \wedge Y:=X \cap Y$ for any $X, Y \in \mathcal{F}$.

Definition. Let $(E, \mathcal{F})$ be a convex geometry with the closure operator $\tau$. The extreme-point operator of $(E, \mathcal{F})$ is an operator ex : $2^{E} \rightarrow 2^{E}$ defined by

$$
\operatorname{ex}(X)=\{e \mid e \in X, e \notin \tau(X \backslash\{e\})\} \quad\left(X \in 2^{E}\right) .
$$

An element in $\operatorname{ex}(X)$ is called an extreme point of $X$. The co-extreme-point operator of $(E, \mathcal{F})$ is an operator ex* $: 2^{E} \rightarrow 2^{E}$ defined by

$$
\operatorname{ex}^{*}(X)=\{e \mid e \in E \backslash \tau(X), \tau(X) \cup\{e\}=\tau(X \cup\{e\})\} \quad\left(X \in 2^{E}\right) .
$$

An element in $\operatorname{ex}^{*}(X)$ is called a co-extreme point of $X$.

For any closed set $X \in \mathcal{F}$ of a convex geometry $(E, \mathcal{F})$, we have

$$
\begin{aligned}
\operatorname{ex}(X) & =\{e \in X \mid X \backslash\{e\} \in \mathcal{F}\}, \\
\operatorname{ex}^{*}(X) & =\{e \in E \backslash X \mid X \cup\{e\} \in \mathcal{F}\}
\end{aligned}
$$

(cf. $[9,(2.5),(2.6)])$.

The extreme-point operator ex : $2^{E} \rightarrow 2^{E}$ satisfies the following properties:

(ex0) $\operatorname{ex}(\{e\})=\{e\}$ for all $e \in E$ (Singleton Identity).

(ex1) $\operatorname{ex}(X) \subseteq X$ for all $X \in 2^{E}$ (Intensionality).

(ex2) $X \subseteq Y \subseteq E \Rightarrow \operatorname{ex}(Y) \cap X \subseteq \operatorname{ex}(X)$ (Chernoff Property).

(ex3) For any $X \in 2^{E}$ and any $p, q \in E \backslash X$, if $p \notin \operatorname{ex}(X \cup\{p\})$ and $q \in \operatorname{ex}(X \cup\{q\})$, then $q \in \operatorname{ex}(X \cup\{p, q\})$.

(ex4) $\operatorname{ex}(Y) \subseteq X \subseteq Y \subseteq E \Rightarrow \operatorname{ex}(X) \subseteq \operatorname{ex}(Y)$ (Aizerman's Axiom).

Note. K. Ando [1] showed that the conditions (ex1)-(ex3) completely characterize the extreme-point operators ex for closure spaces ([1, Theorem 2]), while the conditions (ex0)-(ex2) and (ex4) completely characterize the extreme-point operators ex for convex geometries ([1, Theorem 4]).

\section{$\S 2.3$. Operations on convex geometries}

In this subsection, we consider some operations on convex geometries such as restriction, contraction, and union. 
Proposition 2.2 ([5, Theorem 5.9]). Let $(E, \mathcal{F})$ be a convex geometry and $X$ a closed set. Then the restriction

$$
(E, \mathcal{F}) \mid X:=\left(X, \mathcal{F}^{(X)}\right)
$$

of $(E, \mathcal{F})$ to $X$ is a convex geometry.

Proposition 2.3 ([5, Theorem 5.10]). Let $(E, \mathcal{F})$ be a convex geometry and $X$ a closed set. Then the contraction

$$
(E, \mathcal{F}) / X:=\left(E \backslash X, \mathcal{F}_{(X)}\right)
$$

of $(E, \mathcal{F})$ by $X$ is a convex geometry.

A minor of a convex geometry $(E, \mathcal{F})$ is any convex geometry on a subset $E^{\prime}$ of $E$ obtained by a sequence of restrictions and contractions.

Corollary 2.4 ([5, Corollary 5.11]). Every minor of a convex geometry is a convex geometry.

Note. A forbidden minor characterization of convex geometries is given as follows (see [5, Theorem 5.12]): A closure space $(E, \mathcal{F})$ is a convex geometry if and only if it has no minor isomorphic to $(\{1,2\},\{\emptyset,\{1,2\}\})$.

Proposition 2.5. Let $\left(E_{1}, \mathcal{F}_{1}\right)$ and $\left(E_{2}, \mathcal{F}_{2}\right)$ be convex geometries with $E_{1} \cap E_{2}$ $=\emptyset$. Let

$$
\mathcal{F}_{1} \sqcup \mathcal{F}_{2}:=\left\{X_{1} \cup X_{2} \subseteq E_{1} \cup E_{2} \mid X_{1} \in \mathcal{F}_{1}, X_{2} \in \mathcal{F}_{2}\right\} .
$$

Then $\left(E_{1} \cup E_{2}, \mathcal{F}_{1} \sqcup \mathcal{F}_{2}\right)$ is a convex geometry.

Proof. We show that $\mathcal{F}_{1} \sqcup \mathcal{F}_{2}$ satisfies the properties (CG0), (CG1), and (CG2). Since $\emptyset, E_{1} \in \mathcal{F}_{1}$ and $\emptyset, E_{2} \in \mathcal{F}_{2}$, we have $\emptyset, E_{1} \cup E_{2} \in \mathcal{F}_{1} \sqcup \mathcal{F}_{2}$, and thus (CG0) holds. For any $X_{1} \cup X_{2}, Y_{1} \cup Y_{2} \in \mathcal{F}_{1} \sqcup \mathcal{F}_{2}$, we have $\left(X_{1} \cup X_{2}\right) \cap\left(Y_{1} \cup Y_{2}\right)=$ $\left(X_{1} \cap Y_{1}\right) \cup\left(X_{2} \cap Y_{2}\right) \in \mathcal{F}_{1} \sqcup \mathcal{F}_{2}$ since $E_{1} \cap E_{2}=\emptyset$, and thus (CG1) holds. Take any $X_{1} \cup X_{2} \in \mathcal{F}_{1} \sqcup \mathcal{F}_{2} \backslash\left\{E_{1} \cup E_{2}\right\}$. Note that $X_{1} \neq E_{1}$ or $X_{2} \neq E_{2}$ hold, so we may assume that $X_{2} \neq E_{2}$. Then, by the property (CG2) for $\left(E_{2}, \mathcal{F}_{2}\right)$, there exists $e \in E_{2} \backslash X_{2}$ such that $X_{2} \cup\{e\} \in \mathcal{F}_{2}$. Therefore we have $e \in\left(E_{1} \cup E_{2}\right) \backslash\left(X_{1} \cup X_{2}\right)$ and $X_{1} \cup X_{2} \cup\{e\} \in \mathcal{F}_{1} \sqcup \mathcal{F}_{2}$, and thus (CG2) holds.

Now we consider operations for a subfamily of the family of closed sets of a convex geometry. 
Proposition 2.6. Let $(E, \mathcal{F})$ be a convex geometry with the closure operator $\tau$, $X$ a closed set, and $\mathcal{A}$ a subfamily of $\mathcal{F}$. Suppose that $\mathcal{A}$ satisfies the following property:

- $A \in \mathcal{A} \Rightarrow X \cap A \in \mathcal{A}$.

Then the restriction $\mathcal{A}^{(X)}$ of $\mathcal{A}$ to $X$ is given by

$$
\mathcal{A}^{(X)}=\{X \cap A \mid A \in \mathcal{A}\} .
$$

In particular, we have

$$
\mathcal{F}^{(X)}=\{X \cap Y \mid Y \in \mathcal{F}\}
$$

Proof. Put $\mathcal{Z}:=\{X \cap A \mid A \in \mathcal{A}\}$. We show $\mathcal{A}^{(X)}=\mathcal{Z}$. Take any $A \in \mathcal{A}^{(X)}$. Then $A \in \mathcal{A}$ and $A \subseteq X$. Thus $A=X \cap A \in \mathcal{Z}$. Take any $X \cap A \in \mathcal{Z}$ with $A \in \mathcal{A}$. Then $X \cap A \in \mathcal{A}$ by the assumption and $X \cap A \subseteq X$. Thus we have $X \cap A \in \mathcal{A}^{(X)}$. Hence $\mathcal{A}^{(X)}=\mathcal{Z}$.

Proposition 2.7. Let $(E, \mathcal{F})$ be a convex geometry with the closure operator $\tau$, $X$ a closed set, and $\mathcal{A}$ a subfamily of $\mathcal{F}$. Suppose that $\mathcal{A}$ satisfies the following property:

- $A \in \mathcal{A} \Rightarrow \tau(X \cup A) \in \mathcal{A}$.

Then the upper restriction $\mathcal{A}_{[X]}$ of $\mathcal{A}$ by $X$ is given by

$$
\mathcal{A}_{[X]}=\{\tau(X \cup A) \mid A \in \mathcal{A}\} .
$$

In particular, we have

$$
\mathcal{F}_{[X]}=\{\tau(X \cup Y) \mid Y \in \mathcal{F}\} .
$$

Proof. Put $\mathcal{Z}:=\{\tau(X \cup A) \mid A \in \mathcal{A}\}$. We show $\mathcal{A}_{[X]}=\mathcal{Z}$. Take any $A \in \mathcal{A}_{[X]}$. Then $A \in \mathcal{A} \subseteq \mathcal{F}$ and $A \supseteq X$. Therefore $A=\tau(A)$ and $A=X \cup A$. Thus we have $A=\tau(A)=\tau(X \cup A) \in \mathcal{Z}$. Take any $\tau(X \cup A) \in \mathcal{Z}$ with $A \in \mathcal{A}$. Then $\tau(X \cup A) \in \mathcal{A}$ by the assumption and $\tau(X \cup A) \supseteq X \cup A \supseteq X$ by (cl1). Thus we have $\tau(X \cup A) \in \mathcal{A}_{[X]}$. Hence $\mathcal{A}_{[X]}=\mathcal{Z}$.

Proposition 2.8. Let $(E, \mathcal{F})$ be a convex geometry with the closure operator $\tau$, $X$ a closed set, and $\mathcal{A}$ a subfamily of $\mathcal{F}$. Suppose that $\mathcal{A}$ satisfies the following property:

- $A \in \mathcal{A} \Rightarrow \tau(X \cup A) \in \mathcal{A}$. 
Then the contraction $\mathcal{A}_{(X)}$ of $\mathcal{A}$ by $X$ is given by

$$
\mathcal{A}_{(X)}=\{\tau(X \cup A) \backslash X \mid A \in \mathcal{A}\} .
$$

In particular, we have

$$
\mathcal{F}_{(X)}=\{\tau(X \cup Y) \backslash X \mid Y \in \mathcal{F}\} \text {. }
$$

Proof. This follows from the definition of contraction and Proposition 2.7.

\section{$\S 3$. Matroids on convex geometries (cg-matroids)}

Let $(E, \mathcal{F})$ be a convex geometry on a finite set $E$ with the family $\mathcal{F}$ of closed sets. Let $\tau: 2^{E} \rightarrow \mathcal{F}$ be the closure operator of the convex geometry $(E, \mathcal{F})$, ex $: \mathcal{F} \rightarrow 2^{E}$ the extreme-point operator of $(E, \mathcal{F})$, and ex* $: \mathcal{F} \rightarrow 2^{E}$ the coextreme-point operator of $(E, \mathcal{F})$.

\section{§3.1. Definition}

First, we recall the definition of a cg-matroid.

Definition $([9])$. Let $(E, \mathcal{F})$ be a convex geometry and $\mathcal{B}$ be a subfamily of $\mathcal{F}$. The pair $M=(E, \mathcal{F} ; \mathcal{B})$ is called a matroid on the convex geometry $(E, \mathcal{F})$, or a cg-matroid for short, if $\mathcal{B}$ satisfies the following three properties:

(B0) $\mathcal{B} \neq \emptyset$.

(B1) $B_{1}, B_{2} \in \mathcal{B}, B_{1} \subseteq B_{2} \Rightarrow B_{1}=B_{2}$.

(BM) (Middle Base Property) For any $B_{1}, B_{2} \in \mathcal{B}$ and $X, Y \in \mathcal{F}$ with $B_{1} \supseteq$ $X \subseteq Y \supseteq B_{2}$, there exists $B \in \mathcal{B}$ such that $X \subseteq B \subseteq Y$.

Each element in the family $\mathcal{B}$ is called a base, and $\mathcal{B}=\mathcal{B}(M)$ is called the family of bases of the cg-matroid $M=(E, \mathcal{F} ; \mathcal{B})$.

Let $M=(E, \mathcal{F} ; \mathcal{B})$ be a cg-matroid with a family $\mathcal{B}$ of bases. Let

$$
\begin{aligned}
& \mathcal{I}=\mathcal{I}(M):=\operatorname{Low}(\mathcal{B}) \cap \mathcal{F}, \\
& \mathcal{S}=\mathcal{S}(M):=\operatorname{Upp}(\mathcal{B}) \cap \mathcal{F} .
\end{aligned}
$$

Each element in $\mathcal{I}(M)$ is called an independent set of the cg-matroid $M$, and each element in $\mathcal{S}(M)$ is called a spanning set of the cg-matroid $M$. Note that $\mathcal{B}=\operatorname{Max}(\mathcal{I})=\operatorname{Min}(\mathcal{S})$.

Example 3.1 ([9, Example 3.2]). Let $(E, \mathcal{F})$ be a convex geometry and $k$ be an integer such that $0 \leq k \leq|E|$. Define $\mathcal{B}:=\{X \in \mathcal{F}|| X \mid=k\}$. Then $(E, \mathcal{F} ; \mathcal{B})$ is a cg-matroid, called a $k$-uniform $c g$-matroid. 
Example 3.2 ([13, Example 2.13]). Let $(E, \mathcal{F})$ be a convex shelling in $\mathbb{R}^{d}$. We call a finite set $X$ of points in $\mathbb{R}^{d}$ a simplex if $\operatorname{dim}(\operatorname{Conv}(X))=|X|-1$. Let

$$
\mathcal{I}=\{X \in \mathcal{F}|\operatorname{dim}(\operatorname{Conv}(X))=| X \mid-1\}
$$

be the family of closed sets which are simplices in $\mathbb{R}^{d}$. Then $(E, \mathcal{F} ; \mathcal{I})$ is a cgmatroid, called an affine cg-matroid.

Example 3.3. Let $(E, \mathcal{F})$ be a convex geometry, $X$ a nonempty closed set, and $A$ a set of extreme points of $X$. Let $\mathcal{B}:=\{X \backslash\{e\} \mid e \in A\}$. Then $(E, \mathcal{F} ; \mathcal{B})$ is a cg-matroid.

Proof. It is easy to see that the properties (B0) and (B1) hold. We show the middle base property (BM). Let $Z, Y$ be closed sets such that $X \backslash\left\{e_{1}\right\} \supseteq Z \subseteq Y \supseteq X \backslash\left\{e_{2}\right\}$ where $e_{1}, e_{2} \in A \subseteq \operatorname{ex}(X)$. If $e_{2} \in Z \subseteq Y$, then we have $Z \subseteq X \backslash\left\{e_{1}\right\} \subseteq X=$ $\left(X \backslash\left\{e_{2}\right\}\right) \cup\left\{e_{2}\right\} \subseteq Y$ with $X \backslash\left\{e_{1}\right\} \in \mathcal{B}$. If $e_{2} \notin Z \subseteq X \backslash\left\{e_{1}\right\}$, then we have $Z \subseteq X \backslash\left\{e_{1}, e_{2}\right\} \subseteq X \backslash\left\{e_{2}\right\} \subseteq Y$ with $X \backslash\left\{e_{2}\right\} \in \mathcal{B}$. Therefore (BM) holds, and thus $(E, \mathcal{F} ; \mathcal{B})$ is a cg-matroid.

Example 3.4. Let $(E, \mathcal{F})$ be a convex geometry, $X \in \mathcal{F} \backslash\{E\}$ a closed set, and $A$ a set of co-extreme points of $X$. Let $\mathcal{B}:=\{X \cup\{e\} \mid e \in A\}$. Then $(E, \mathcal{F} ; \mathcal{B})$ is a cg-matroid.

Proof. It is easy to see that the properties (B0) and (B1) hold. We show the middle base property (BM). Let $Z, Y$ be closed sets such that $X \cup\left\{e_{1}\right\} \supseteq Z \subseteq Y \supseteq X \cup\left\{e_{2}\right\}$ where $e_{1}, e_{2} \in A \subseteq \operatorname{ex}^{*}(X)$. If $e_{1} \in Z \subseteq Y$, then we have $Z \subseteq X \cup\left\{e_{1}\right\} \subseteq$ $\left(X \cup\left\{e_{2}\right\}\right) \cup\left\{e_{1}\right\} \subseteq Y$ with $X \cup\left\{e_{1}\right\} \in \mathcal{B}$. If $e_{1} \notin Z \subseteq X \cup\left\{e_{1}\right\}$, then we have $Z \subseteq X \subseteq X \cup\left\{e_{2}\right\} \subseteq Y$ with $X \cup\left\{e_{2}\right\} \in \mathcal{B}$. Therefore (BM) holds, and thus $(E, \mathcal{F} ; \mathcal{B})$ is a cg-matroid.

\section{§3.2. Combinatorial structure of cg-matroids}

The family of bases of a cg-matroid satisfies the following.

Theorem 3.5 ([9, Theorem 3.3]). All the bases in a cg-matroid have the same cardinality, i.e., for any cg-matroid $(E, \mathcal{F} ; \mathcal{B})$, the following property holds:

(B1) ${ }^{\prime} B_{1}, B_{2} \in \mathcal{B} \Rightarrow\left|B_{1}\right|=\left|B_{2}\right|$.

In [9], S. Fujishige, G. A. Koshevoy, and Y. Sano have given a characterization of the family of bases of a cg-matroid by an "exchange property" as follows.

Theorem 3.6 ([9, Theorem 3.7]). Let $(E, \mathcal{F})$ be a convex geometry and $\mathcal{B}$ a subfamily of $\mathcal{F}$. Then $\mathcal{B}$ is the family of bases of a cg-matroid on $(E, \mathcal{F})$ if and only if $\mathcal{B}$ satisfies $(\mathrm{B} 0)$ and $(\mathrm{BE})$, where 
(BE) (Exchange Property) For any $B_{1}, B_{2} \in \mathcal{B}$ and any $e_{1} \in \operatorname{ex}\left(\tau\left(B_{1} \cup B_{2}\right)\right) \backslash B_{2}$, there exists $e_{2} \in \tau\left(B_{1} \cup B_{2}\right) \backslash B_{1}$ such that $\left(B_{1} \backslash\left\{e_{1}\right\}\right) \cup\left\{e_{2}\right\} \in \mathcal{B}$.

Example 3.7. Let $(E, \mathcal{F})$ be the convex shelling of the 12 points in the plane given in Figure 1. Recall that for a convex shelling $(E, \mathcal{F})$, the closure $\tau(X)$ of a subset $X$ of $E$ is the set of points of $E$ which are contained in the convex hull of $X$, and that an extreme point of a closed set $Y$ is a vertex of the convex hull of $Y$.

Let $M=(E, \mathcal{F} ; \mathcal{B})$ be the 4 -uniform cg-matroid defined on $(E, \mathcal{F})$. Take two bases $B_{1}:=\{2,5,6,10\} \in \mathcal{B}$ and $B_{2}:=\{4,7,8,10\} \in \mathcal{B}$ of the cg-matroid $M$. Then the closure $\tau\left(B_{1} \cup B_{2}\right)$ of the union of the bases $B_{1}$ and $B_{2}$ is $\{2,3,4,5,6,7,8,10\}$, and the set of extreme points of $\tau\left(B_{1} \cup B_{2}\right)$ is $\{2,4,5,8,10\}$. So we have

$$
\operatorname{ex}\left(\tau\left(B_{1} \cup B_{2}\right)\right) \backslash B_{2}=\{2,5\}, \quad \tau\left(B_{1} \cup B_{2}\right) \backslash B_{1}=\{3,4,7,8\} .
$$

The elements $e_{1}$ and $e_{2}$ in the statement of the exchange property (BE) are taken from the above sets. Note that the element 3 is not in the base $B_{2}$.

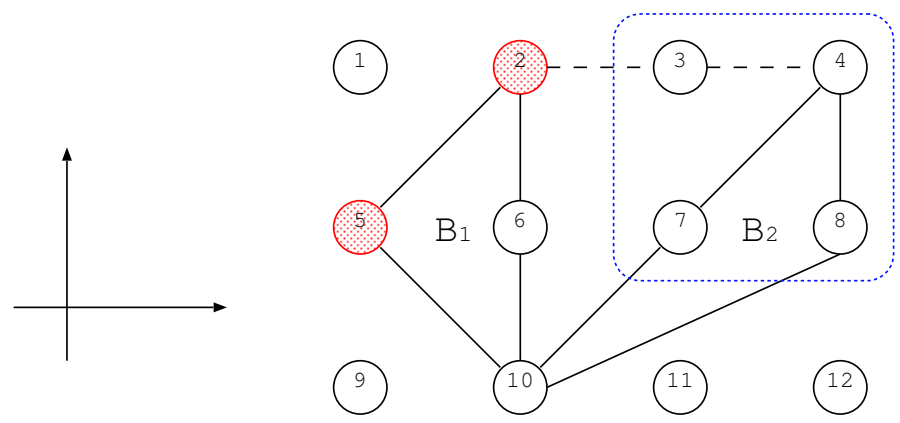

Figure 1. The 4-uniform cg-matroid defined on a convex shelling of 12 points in the plane.

The authors of [9] have also given a characterization of the family of independent sets of a cg-matroid.

Theorem 3.8 ([9, Theorems 3.10, 3.12]). Let $(E, \mathcal{F})$ be a convex geometry and $\mathcal{I}$ a subfamily of $\mathcal{F}$. Then $\mathcal{I}$ is the family of independent sets of a cg-matroid on $(E, \mathcal{F})$ if and only if $\mathcal{I}$ satisfies the following properties:

(I0) $\emptyset \in \mathcal{I}$.

(I1) $I_{1} \in \mathcal{F}, I_{2} \in \mathcal{I}, I_{1} \subseteq I_{2} \Rightarrow I_{1} \in \mathcal{I}$.

(IA) (Augmentation Property) For any $I_{1} \in \mathcal{I}$ and $I_{2} \in \operatorname{Max}(\mathcal{I})$ with $\left|I_{1}\right|<\left|I_{2}\right|$, there exists $e \in \tau\left(I_{1} \cup I_{2}\right) \backslash I_{1}$ such that $I_{1} \cup\{e\} \in \mathcal{I}$. 
By Theorem 3.8, we call the pair $(E, \mathcal{F} ; \mathcal{I})$ of a convex geometry $(E, \mathcal{F})$ and a subfamily $\mathcal{I}$ of $\mathcal{F}$ a cg-matroid with a family $\mathcal{I}$ of independent sets if $\mathcal{I}$ satisfies the properties (I0), (I1), and (IA).

Proposition 3.9. The property (I1) is equivalent to the following property:

(I1)' (Intersection Property) $X \in \mathcal{F}, I \in \mathcal{I} \Rightarrow X \cap I \in \mathcal{I}$.

Proof. Suppose that the property (I1) holds. Take any $X \in \mathcal{F}$ and $I \in \mathcal{I} \subseteq \mathcal{F}$. Then we have $X \cap I \in \mathcal{F}$ by the property (CG1) and $X \cap I \subseteq I \in \mathcal{I}$. So we have $X \cap I \in \mathcal{I}$ by (I1), and thus the property (I1)' holds.

Conversely, suppose that (I1)' holds. Take any $I_{1} \in \mathcal{F}$ and $I_{2} \in \mathcal{I}$ with $I_{1} \subseteq I_{2}$. Then we have $I_{1}=I_{1} \cap I_{2} \in \mathcal{I}$ by (I1)', and thus the property (I1) holds.

Proposition 3.10. Let $M=(E, \mathcal{F} ; \mathcal{I})$ be a cg-matroid with a family $\mathcal{I}$ of independent sets and $X$ a closed set. Then the restriction $\mathcal{I}^{(X)}=\{I \in \mathcal{I} \mid I \subseteq X\}$ of $\mathcal{I}$ to $X$ is given by

$$
\mathcal{I}^{(X)}=\{X \cap I \mid I \in \mathcal{I}\}
$$

Proof. This follows from Propositions 2.6 and 3.9.

Proposition 3.11. The augmentation property (IA) is equivalent to the following property:

(IA $)^{\prime}$ For any $I_{1}, I_{2} \in \mathcal{I}$ with $\left|I_{1}\right|<\left|I_{2}\right|$ such that $I \subseteq \tau\left(I_{1} \cup I_{2}\right)$ for some $I \in$ $\operatorname{Max}(\mathcal{I})$, there exists $e \in \tau\left(I_{1} \cup I_{2}\right) \backslash I_{1}$ such that $I_{1} \cup\{e\} \in \mathcal{I}$.

Proof. It is easy to see that the property (IA)' implies (IA). Conversely, suppose that the property (IA) holds. Take $I_{1}, I_{2} \in \mathcal{I}$ with $\left|I_{1}\right|<\left|I_{2}\right|$ such that $I \subseteq \tau\left(I_{1} \cup I_{2}\right)$ for some $I \in \operatorname{Max}(\mathcal{I})$. Since $I_{1} \subseteq I_{1} \cup I_{2} \subseteq \tau\left(I_{1} \cup I_{2}\right)$, we have $I_{1} \cup I \subseteq \tau\left(I_{1} \cup I_{2}\right)$. Then $\tau\left(I_{1} \cup I\right) \subseteq \tau\left(\tau\left(I_{1} \cup I_{2}\right)\right)=\tau\left(I_{1} \cup I_{2}\right)$ by (cl2) and (cl3). By the property (IA), there exists $e \in \tau\left(I_{1} \cup I\right) \backslash I_{1} \subseteq \tau\left(I_{1} \cup I_{2}\right) \backslash I_{1}$ such that $I_{1} \cup\{e\} \in \mathcal{I}$. Hence the property $(\mathrm{IA})^{\prime}$ holds.

In the following, we give a characterization of the family of spanning sets of a cg-matroid.

Theorem 3.12. The family $\mathcal{S}=\mathcal{S}(M)$ of spanning sets of a cg-matroid $M=$ $(E, \mathcal{F} ; \mathcal{B})$ with a family $\mathcal{B}$ of bases satisfies the following properties.

(S0) $E \in \mathcal{S}$.

(S1) $S_{1} \in \mathcal{F}, S_{2} \in \mathcal{S}, S_{1} \supseteq S_{2} \Rightarrow S_{1} \in \mathcal{S}$.

(SR) (Reduction Property) For any $S_{1} \in \mathcal{S}$ and $S_{2} \in \operatorname{Min}(\mathcal{S})$ with $\left|S_{1}\right|>\left|S_{2}\right|$, there exists $e \in S_{1} \backslash S_{2}$ such that $S_{1} \backslash\{e\} \in \mathcal{S}$. 
Proof. We can easily check from the property (B0) and the definition of spanning sets that (S0) and (S1) hold. Let us show that the reduction property (SR) holds. For any $S_{1} \in \mathcal{S} \subseteq \mathcal{F}$ and $S_{2} \in \operatorname{Min}(\mathcal{S}) \subseteq \mathcal{F}$ with $\left|S_{1}\right|>\left|S_{2}\right|$, there exists a base $B_{1} \in \mathcal{B}$ such that $B_{1} \subsetneq S_{1}$, and $S_{2}$ itself is a base because of its minimality. Therefore $S_{2} \supseteq S_{1} \cap S_{2} \subseteq S_{1} \supsetneq B_{1}$. Note that $S_{1} \cap S_{2} \in \mathcal{F}$ by the property (CG1). Hence, by the middle base property (BM), there exists a base $B \in \mathcal{B}$ such that $S_{1} \cap S_{2} \subseteq B \subseteq S_{1}$. Note that $S_{1} \notin \mathcal{B}$ by the property (B1)', since $|B|=\left|S_{2}\right|<\left|S_{1}\right|$. Therefore $B \neq S_{1}$. By considering a chain of closed sets of $(E, \mathcal{F})$ containing $B$ and $S_{1}$, we can take $e \in S_{1} \backslash B \subseteq S_{1} \backslash S_{2}$ such that $B \subseteq S_{1} \backslash\{e\}$. Hence the reduction property (SR) holds.

Conversely, we can show the following.

Theorem 3.13. Let $(E, \mathcal{F})$ be a convex geometry and $\mathcal{S}$ a subfamily of $\mathcal{F}$. Suppose that the family $\mathcal{S}$ satisfies the properties $(\mathrm{S} 0),(\mathrm{S} 1)$, and $(\mathrm{SR})$. Put $\mathcal{B}:=\operatorname{Min}(\mathcal{S})$. Then $\mathcal{B}$ is the family of bases of a cg-matroid on $(E, \mathcal{F})$.

Before proving this theorem, we show the following lemma.

Lemma 3.14. Let $(E, \mathcal{F})$ be a convex geometry and $\mathcal{S} \subseteq \mathcal{F}$ a subfamily of $\mathcal{F}$. Suppose that the family $\mathcal{S}$ satisfies the properties (S0), (S1), and (SR). Then the family $\mathcal{B}=\operatorname{Min}(\mathcal{S})$ satisfies the property $(\mathrm{B} 1)^{\prime}$.

Proof. If we have $\left|B_{1}\right|>\left|B_{2}\right|$ for some $B_{1}, B_{2} \in \mathcal{B}=\operatorname{Min}(\mathcal{S})$, then from the reduction property (SR) there exists $e \in B_{1} \backslash B_{2}$ such that $B_{1} \backslash\{e\} \in \mathcal{S}$, which contradicts the minimality of $B_{1}$ in $\mathcal{S}$.

Proof of Theorem 3.13. The property (B0) follows from the property (S0) and the definition of a spanning set. The property (B1) follows from Lemma 3.14.

Now, we show that the middle base property (BM) holds. For a nonnegative integer $k$, we consider the following property:

$(\mathrm{BM})_{k}$ For any $B_{1}, B_{2} \in \mathcal{B}$ and $X, Y \in \mathcal{F}$ with $B_{1} \supseteq X \subseteq Y \supseteq B_{2}$ and $k=$ $\left|Y \backslash B_{1}\right|$, there exists $B \in \mathcal{B}$ such that $X \subseteq B \subseteq Y$.

Note that the middle base property (BM) holds if and only if the property $(\mathrm{BM})_{k}$ holds for any $k \in \mathbb{Z}_{\geq 0}$. So we show that (BM) $)_{k}$ holds for any $k \in \mathbb{Z}_{\geq 0}$ by induction on $k$. When $k=0$, we have $B_{2} \subseteq Y \subseteq B_{1}$. It follows from this fact and the property (B1) that $X \subseteq B_{1}=B_{2}=Y$, and thus the property (BM) $)_{0}$ holds. Next, assume that $(\mathrm{BM})_{k}$ holds for some $k \geq 0$. Suppose $\left|Y \backslash B_{1}\right|=k+1$. If $B_{2}=Y$, then $X \subseteq B_{2}=Y$, and the property $(\mathrm{BM})_{k+1}$ holds. So we assume that $B_{2} \subsetneq Y$. Since $Y \in \mathcal{S}, B_{1}$ is minimal in $\mathcal{S}$, and $|Y|>\left|B_{1}\right|\left(=\left|B_{2}\right|\right)$, it follows from the reduction 
property (SR) that there exists $e \in Y \backslash B_{1}$ such that $Y^{\prime}:=Y \backslash\{e\} \in \mathcal{S}$. Then there exists a base $B_{2}^{\prime} \in \mathcal{B}$ such that $B_{2}^{\prime} \subseteq Y^{\prime}$. Note that $\left|Y^{\prime} \backslash B_{1}\right|=\left|Y \backslash B_{1}\right|-1=k$ and $X \subseteq Y^{\prime}$ since $e \in Y$ and $e \notin B_{1} \supseteq X$. By the property $(\mathrm{BM})_{k}$, there exists $B \in \mathcal{B}$ such that $X \subseteq B \subseteq Y^{\prime} \subsetneq Y$. Thus the property $(\mathrm{BM})_{k+1}$ holds.

By Theorems 3.12 and 3.13 , we call the pair $(E, \mathcal{F} ; \mathcal{S})$ of a convex geometry $(E, \mathcal{F})$ and a subfamily $\mathcal{S}$ of $\mathcal{F}$ a $c$-matroid with a family $\mathcal{S}$ of spanning sets if $\mathcal{S}$ satisfies the properties (S0), (S1), and (SR).

Proposition 3.15. The property (S1) is equivalent to the following property:

$(\mathrm{S} 1)^{\prime} \quad X \in \mathcal{F}, S \in \mathcal{S} \Rightarrow \tau(X \cup S) \in \mathcal{S}$.

Proof. Suppose that the property (S1) holds. Take any $X \in \mathcal{F}$ and $S \in \mathcal{S}$. Then we have $\tau(X \cup S) \in \mathcal{F}$ and $\tau(X \cup S) \supseteq X \cup S \supseteq S \in \mathcal{S}$. So $\tau(X \cup S) \in \mathcal{S}$ by (S1), and thus the property $(\mathrm{S} 1)^{\prime}$ holds.

Suppose that the property $(\mathrm{S} 1)^{\prime}$ holds. Take any $S_{1} \in \mathcal{F}$ and $S_{2} \in \mathcal{S}$ with $S_{1} \supseteq S_{2}$. Then $S_{1}=\tau\left(S_{1}\right)$ and $S_{1}=S_{1} \cup S_{2}$. Therefore we have $S_{1}=\tau\left(S_{1}\right)=$ $\tau\left(S_{1} \cup S_{2}\right) \in \mathcal{S}$ by $(\mathrm{S} 1)^{\prime}$, and thus the property (S1) holds.

Proposition 3.16. Let $M=(E, \mathcal{F} ; \mathcal{S})$ be a cg-matroid with a family $\mathcal{S}$ of spanning sets and $X$ a closed set. Then the contraction $\mathcal{S}_{(X)}=\{S \backslash X \mid S \in \mathcal{S}, S \supseteq X\}$ of $\mathcal{S}$ by $X$ is given by

$$
\mathcal{S}_{(X)}=\{\tau(X \cup S) \backslash X \mid S \in \mathcal{S}\}
$$

Proof. This follows from Propositions 2.8 and 3.15 .

Proposition 3.17. The reduction property (SR) is equivalent to the following property:

$(\mathrm{SR})^{\prime}$ For any $S_{1}, S_{2} \in \mathcal{S}$ with $\left|S_{1}\right|>\left|S_{2}\right|$ such that $S_{1} \cap S_{2} \subseteq S$ for some $S \in$ $\operatorname{Min}(\mathcal{S})$, there exists $e \in S_{1} \backslash S_{2}$ such that $S_{1} \backslash\{e\} \in \mathcal{S}$.

Proof. It is easy to see that the property (SR)' implies (SR). Conversely, suppose that the property (SR) holds. Take $S_{1}, S_{2} \in \mathcal{S}$ with $\left|S_{1}\right|>\left|S_{2}\right|$ such that $S_{1} \cap S_{2} \subseteq$ $S$ for some $S \in \operatorname{Min}(\mathcal{S})$. Then $S_{1} \cap S_{2} \subseteq S_{1} \cap S$. By the property (SR), there exists $e \in S_{1} \backslash S \subseteq S_{1} \backslash S_{2}$ such that $S_{1} \backslash\{e\} \in \mathcal{S}$. Hence the property (SR)' holds.

\section{$\S 4$. Subclasses of cg-matroids}

\section{§4.1. Strict cg-matroids}

In this subsection, we discuss strict cg-matroids. First, we recall the definition. 
Definition $([9])$. Let $(E, \mathcal{F})$ be a convex geometry and $\mathcal{I}$ be a subfamily of $\mathcal{F}$. We call $(E, \mathcal{F} ; \mathcal{I})$ a strict cg-matroid with a family $\mathcal{I}$ of independent sets if $\mathcal{I}$ satisfies the properties (I0), (I1), and the strict augmentation property (IsA), where

(IsA) (Strict Augmentation Property) For any $I_{1}, I_{2} \in \mathcal{I}$ with $\left|I_{1}\right|<\left|I_{2}\right|$, there exists $e \in \tau\left(I_{1} \cup I_{2}\right) \backslash I_{1}$ such that $I_{1} \cup\{e\} \in \mathcal{I}$.

By definition, any strict cg-matroid is a cg-matroid. It should also be noted that in the case of matroids, i.e., when $\mathcal{F}=2^{E}$, the set of axioms (I0), (I1), and (IA) and that of (I0), (I1), and (IsA) are equivalent. But in the case of cg-matroids they are not equivalent; the following example shows a cg-matroid that is not a strict cg-matroid.

Example 4.1 ([9, Example 4.2]). Let $(E, \mathcal{F})$ be the convex shelling of the five points in the plane given in Figure 2 (left), i.e., $E=\{1,2,3,4,5\}$ and $\mathcal{F}=$ $2^{E} \backslash\{\{1,2,4,5\},\{1,2,4\},\{1,2,5\}\}$. Let $\mathcal{B}=\{\{1,2,3\},\{2,4,5\},\{2,3,4\},\{2,3,5\}\}$. Then $(E, \mathcal{F} ; \mathcal{B})$ is a cg-matroid with a family of bases. But it is not a strict $\mathrm{cg}$ matroid. To see this, let $I_{1}=\{1\}$ and $I_{2}=\{4,5\}$. Then $I_{1}$ and $I_{2}$ are independent sets of the cg-matroid. Since $\left|I_{1}\right|<\left|I_{2}\right|$ and $\tau\left(I_{1} \cup I_{2}\right) \backslash I_{1}=\{4,5\}$, the strict augmentation property (IsA) implies that $\{1,4\}$ or $\{1,5\}$ should be an independent set. But neither $\{1,4\}$ nor $\{1,5\}$ is included in any member of $\mathcal{B}$. Hence the present $\mathrm{cg}$-matroid does not satisfy the strict augmentation property (IsA).
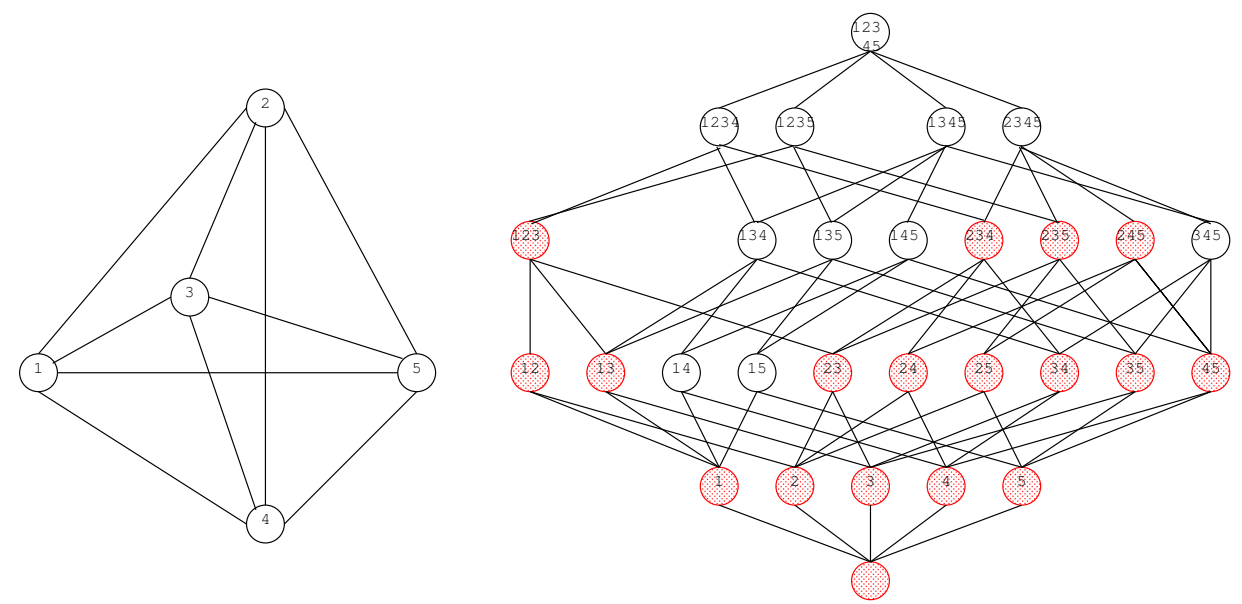

Figure 2. A non-strict cg-matroid.

Example 4.2. Any uniform cg-matroid is a strict cg-matroid.

Example 4.3. Any affine cg-matroid is a strict cg-matroid. 
Example 4.4. Any cg-matroid $(E, \mathcal{F} ; \mathcal{B})$ with the property $|B| \leq 2$ for all $B \in \mathcal{B}$ is a strict cg-matroid.

Strict cg-matroids are characterized by the following properties.

Theorem 4.5 ([9, Theorem 4.4, Lemmas 4.5, 4.6]). Let $(E, \mathcal{F})$ be a convex geometry and $\mathcal{I}$ a subfamily of $\mathcal{F}$. Suppose that $\mathcal{I}$ satisfies the properties (I0) and (I1). Then the strict augmentation property (IsA) is equivalent to each of the following properties.

(ILA) (Local Augmentation Property) For any $I_{1}, I_{2} \in \mathcal{I}$ with $\left|I_{1}\right|+1=\left|I_{2}\right|$, there exists $e \in \tau\left(I_{1} \cup I_{2}\right) \backslash I_{1}$ such that $I_{1} \cup\{e\} \in \mathcal{I}$.

(IS) (Steinitz Exchange Property) For each $X \in \mathcal{F}$, all the maximal elements of $\mathcal{I}^{(X)}$ have the same cardinality.

A characterization of strict cg-matroids in terms of the family of bases is as follows.

Theorem 4.6. Let $M=(E, \mathcal{F} ; \mathcal{B})$ be a cg-matroid with a family $\mathcal{B}$ of bases. Then $M$ is a strict cg-matroid if and only if $\mathcal{B}$ satisfies the following property:

(BS) For each $X \in \mathcal{F}$, all the maximal elements of $\{X \cap B \mid B \in \mathcal{B}\}$ have the same cardinality.

Proof. Since $\operatorname{Max}\{X \cap B \mid B \in \mathcal{B}(M)\}=\operatorname{Max}\{X \cap I \mid I \in \mathcal{I}:=\mathcal{I}(M)\}=$ $\operatorname{Max}\left(\mathcal{I}^{(X)}\right)$, the properties (IS) and (BS) are equivalent for $M$. Thus the assertion follows from Theorem 4.5.

By Theorem 4.6, we call $(E, \mathcal{F} ; \mathcal{B})$ a strict $c$-matroid with a family $\mathcal{B}$ of bases if $\mathcal{B}$ satisfies the properties (B0), (B1), (BM), and (BS).

\section{$\S 4.2$. Co-strict cg-matroids}

Let us consider the following reduction property that is stronger than (SR) given in Theorem 3.12. Note that we do not require that $S_{2}$ is minimal in $\mathcal{S}$.

Definition. Let $(E, \mathcal{F})$ be a convex geometry and $\mathcal{S}$ be a subfamily of $\mathcal{F}$. We call $(E, \mathcal{F} ; \mathcal{S})$ a co-strict cg-matroid with a family $\mathcal{S}$ of spanning sets if the family $\mathcal{S}$ satisfies the properties (S0), (S1), and the strict reduction property (SsR), where

(SsR) (Strict Reduction Property) For any $S_{1}, S_{2} \in \mathcal{S}$ with $\left|S_{1}\right|>\left|S_{2}\right|$, there exists $e \in S_{1} \backslash S_{2}$ such that $S_{1} \backslash\{e\} \in \mathcal{S}$.

By definition, any co-strict cg-matroid is a cg-matroid. It should also be noted that in the case of matroids, i.e., when $\mathcal{F}=2^{E}$, the set of axioms (S0), (S1), and $(\mathrm{SR})$ and that of $(\mathrm{S} 0),(\mathrm{S} 1)$, and $(\mathrm{SsR})$ are equivalent. But in the case of cg- 
matroids they are not equivalent; the following example shows a cg-matroid that is not a co-strict cg-matroid.

Example 4.7. Let $(E, \mathcal{F})$ be the convex shelling of the five points in the plane given in Figure 3 (left), i.e., $E=\{1,2,3,4,5\}$ and $\mathcal{F}=2^{E} \backslash\{\{4,5\},\{1,3,4\}$, $\{1,4,5\},\{2,4,5\},\{1,2,4,5\},\{1,3,4,5\}\}$. Let $\mathcal{B}=\{\{3,4\},\{3,5\}\}$. Then $(E, \mathcal{F} ; \mathcal{B})$ is a cg-matroid with a family of bases. But it is not a co-strict cg-matroid. To see this, let $S_{1}=\{1,2,3,4\}$ and $S_{2}=\{1,3,5\}$. Then $S_{1}$ and $S_{2}$ are spanning sets of the cg-matroid. Since $\left|S_{1}\right|>\left|S_{2}\right|$ and $S_{1} \backslash S_{2}=\{2,4\}$, the strict reduction property (SsR) implies that $\{1,3,4\}$ or $\{2,3,4\}$ should be a spanning set. But we have $\{1,3,4\} \notin \mathcal{F}$, and $\{2,3,4\}$ does not contain any member of $\mathcal{B}$. Hence the present cg-matroid does not satisfy the strict reduction property (SsR).
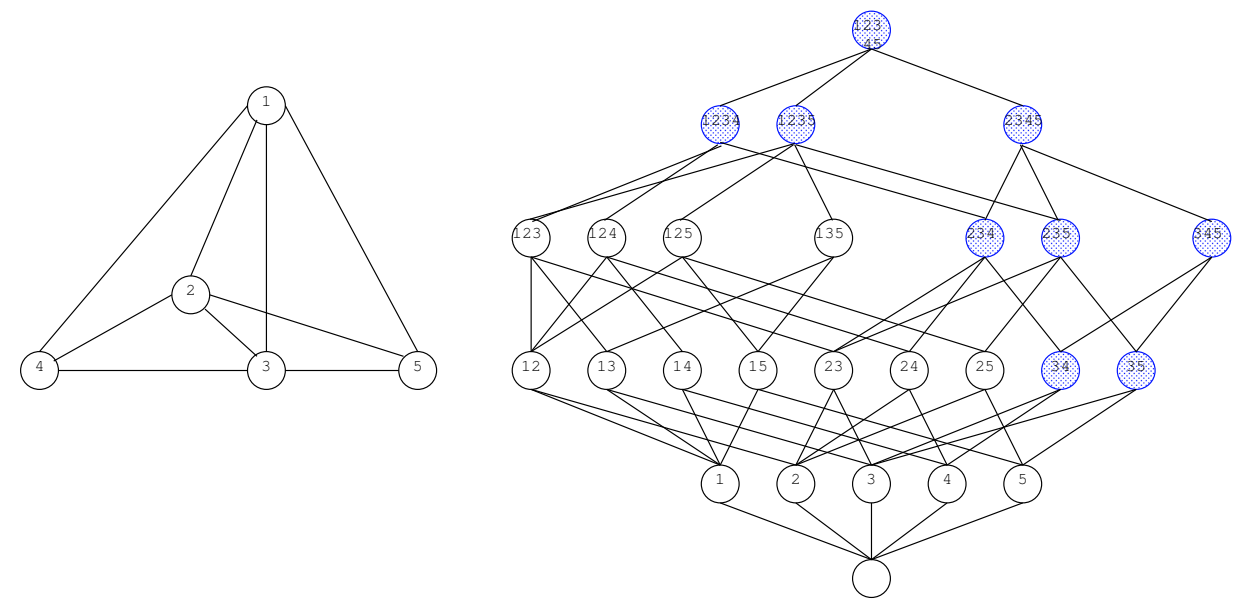

Figure 3. A non-co-strict cg-matroid.

Example 4.8. Any uniform cg-matroid is a co-strict cg-matroid.

Example 4.9. Any cg-matroid $(E, \mathcal{F} ; \mathcal{B})$ with the property $|B| \geq|E|-2$ for all $B \in \mathcal{B}$ is a co-strict cg-matroid.

First, we show the following characterization.

Theorem 4.10. Let $(E, \mathcal{F})$ be a convex geometry and $\mathcal{S}$ a subfamily of $\mathcal{F}$. Suppose that the family $\mathcal{S}$ satisfies the properties $(\mathrm{S} 0)$ and $(\mathrm{S} 1)$. Then $(E, \mathcal{F} ; \mathcal{S})$ is a costrict cg-matroid with a family $\mathcal{S}$ of spanning sets if and only if $\mathcal{S}$ satisfies the following property:

(SLR) (Local Reduction Property) For any $S_{1}, S_{2} \in \mathcal{S}$ with $\left|S_{1}\right|=\left|S_{2}\right|+1$, there exists $e \in S_{1} \backslash S_{2}$ such that $S_{1} \backslash\{e\} \in \mathcal{S}$. 
Proof. The implication (SsR) $\Rightarrow(\mathrm{SLR})$ is trivial. We show the converse, (SLR) $\Rightarrow$ (SsR). Consider $S_{1}, S_{2} \in \mathcal{S}$ with $\left|S_{1}\right|>\left|S_{2}\right|$. Then there exists a closed set $S$ such that $S_{2} \subseteq S$ and $|S|=\left|S_{1}\right|-1$ by the property (CG2). From the property (S1), we have $S \in \mathcal{S}$. Therefore, from the local reduction property (SLR), there exists $e \in S_{1} \backslash S$ such that $S_{1} \backslash\{e\} \in \mathcal{S}$. Since $S_{2} \subseteq S$, we have $S_{1} \backslash S \subseteq S_{1} \backslash S_{2}$, and thus $e \in S_{1} \backslash S_{2}$. Hence the strict reduction property (SsR) holds.

Theorem 4.11. Let $(E, \mathcal{F})$ be a convex geometry and $\mathcal{S}$ a subfamily of $\mathcal{F}$. Suppose that $\mathcal{S}$ satisfies the properties $(\mathrm{S} 0)$ and $(\mathrm{S} 1)$. Then $(E, \mathcal{F} ; \mathcal{S})$ is a co-strict cgmatroid with a family $\mathcal{S}$ of spanning sets if and only if $\mathcal{S}$ satisfies the following property:

(SS) For each $X \in \mathcal{F}$, all the minimal elements of $\mathcal{S}_{[X]}$ have the same cardinality.

Proof. First, we show the "only if" part. Take any $X \in \mathcal{F}$. Note that $\mathcal{S}_{[X]}=$ $\{S \in \mathcal{S} \mid S \supseteq X\}=\{\tau(X \cup S) \mid S \in \mathcal{S}\}$ by Propositions 2.7 and 3.15. Suppose that $\tau\left(X \cup S_{1}\right)$ and $\tau\left(X \cup S_{2}\right)$ are minimal in $\mathcal{S}_{[X]}$ and $\left|\tau\left(X \cup S_{1}\right)\right|>\left|\tau\left(X \cup S_{2}\right)\right|$ where $S_{1}, S_{2} \in \mathcal{S}$. Then it follows from the property (S1) that $\tau\left(X \cup S_{1}\right) \in \mathcal{S}$ and $\tau\left(X \cup S_{2}\right) \in \mathcal{S}$ since $\tau\left(X \cup S_{i}\right) \in \mathcal{F}$ and $S_{i} \subseteq \tau\left(X \cup S_{i}\right)$ for $i=1,2$. Therefore, from the strict reduction property (SsR), there exists $e \in \tau\left(X \cup S_{1}\right) \backslash \tau\left(X \cup S_{2}\right)$ such that $\tau\left(X \cup S_{1}\right) \backslash\{e\} \in \mathcal{S}$. Note that $\tau\left(X \cup S_{1}\right) \backslash \tau\left(X \cup S_{2}\right) \subseteq \tau\left(X \cup S_{1}\right) \backslash X$ since $X \subseteq X \cup S_{2} \subseteq \tau\left(X \cup S_{2}\right)$, and therefore $e \notin X$. Then $\tau\left(X \cup S_{1}\right) \backslash\{e\} \supseteq X$ since $e \notin X$. Thus we have $\tau\left(X \cup S_{1}\right) \backslash\{e\} \in \mathcal{S}_{[X]}$, which contradicts the minimality of $\tau\left(X \cup S_{1}\right)$ in $\mathcal{S}_{[X]}$.

Next, we show the "if" part. Suppose that $S_{1}, S_{2} \in \mathcal{S}$ and $\left|S_{1}\right|>\left|S_{2}\right|$. Consider $X=S_{1} \cap S_{2}$ in the property (SS). Note that $S_{1} \cap S_{2} \in \mathcal{F}$ by the property (CG1). Then $S_{i} \in \mathcal{S}_{\left[S_{1} \cap S_{2}\right]}$ for $i=1,2$. From the property (SS) and the assumption that $\left|S_{1}\right|>\left|S_{2}\right|, S_{1}$ is not minimal in $\mathcal{S}_{\left[S_{1} \cap S_{2}\right]}$. Hence, there exists $e \in S_{1} \backslash\left(S_{1} \cap S_{2}\right)=$ $S_{1} \backslash S_{2}$ such that $S_{1} \backslash\{e\} \in \mathcal{S}_{\left[S_{1} \cap S_{2}\right]} \subseteq \mathcal{S}$. Hence the strict reduction property (SsR) holds.

A characterization of co-strict cg-matroids in terms of the family of bases is given as follows.

Theorem 4.12. Let $M=(E, \mathcal{F} ; \mathcal{B})$ be a cg-matroid with a family $\mathcal{B}$ of bases. Then $M$ is a co-strict cg-matroid if and only if $\mathcal{B}$ satisfies the following property:

$(\mathrm{BcS})$ For each $X \in \mathcal{F}$, all the minimal elements of $\{\tau(X \cup B) \mid B \in \mathcal{B}\}$ have the same cardinality.

Proof. Since $\operatorname{Min}\{\tau(X \cup B) \mid B \in \mathcal{B}(M)\}=\operatorname{Min}\{\tau(X \cup S) \mid S \in \mathcal{S}:=\mathcal{S}(M)\}=$ $\operatorname{Min}\left(\mathcal{S}_{[X]}\right)$, the properties (SS) and $(\mathrm{BcS})$ are equivalent for $M$. Thus the assertion follows from Theorem 4.11. 
By Theorem 4.12 , we call $(E, \mathcal{F} ; \mathcal{B})$ a co-strict cg-matroid with a family $\mathcal{B}$ of bases if $\mathcal{B}$ satisfies the properties (B0), (B1), (BM), and (BcS).

\section{$\S 4.3$. Tame and wild cg-matroids}

Definition. A cg-matroid is called tame if it is both a strict cg-matroid and a co-strict cg-matroid. A cg-matroid is called wild if it is neither strict nor co-strict.

Example 4.13. Any cg-matroid defined on a poset shelling (i.e. a poset matroid) is a tame cg-matroid.

Example 4.14. Any cg-matroid with a single base is a tame cg-matroid.

Example 4.15. Let $(E, \mathcal{F})$ be a convex geometry with $|E| \leq 5$. Then no cgmatroid $M$ on $(E, \mathcal{F})$ is wild, i.e., every cg-matroid $M$ is either strict or co-strict.

Proof. This follows from Examples 4.4 and 4.9.

Example 4.16. Let $\left(E^{\prime}, \mathcal{F}^{\prime}\right)$ be the convex shelling of the seven points in $\mathbb{R}^{3}$ given in Figure 4, i.e., $E^{\prime}=E \cup\{6,7\}$ and $\mathcal{F}^{\prime}=\{X \cup A \mid X \in \mathcal{F}, A \subseteq\{6,7\}\} \backslash$ $\{\{1,2,3,7\}\}$, where $(E, \mathcal{F})$ is the convex shelling given in Example 4.1. Let $\mathcal{B}=$

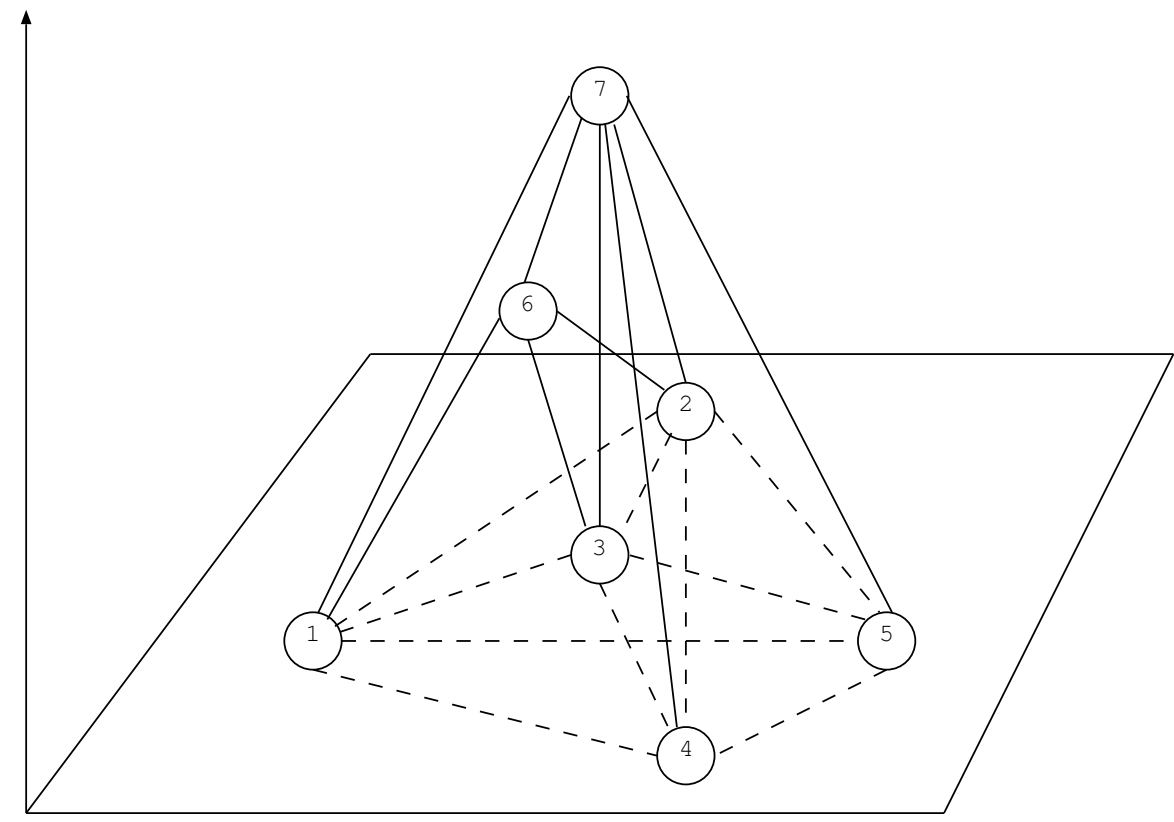

Figure 4. A convex shelling of seven points in $\mathbb{R}^{3}$. 
$\{\{1,2,3\},\{2,3,4\},\{2,3,5\},\{2,4,5\}\}$. Then $\left(E^{\prime}, \mathcal{F}^{\prime} ; \mathcal{B}\right)$ is a wild cg-matroid with a family of bases.

To see this, let $I_{1}=\{2\}$ and $I_{2}=\{3,4\}$. Then $I_{1}$ and $I_{2}$ are independent sets of the cg-matroid. Since $\left|I_{1}\right|<\left|I_{2}\right|$ and $\tau\left(I_{1} \cup I_{2}\right) \backslash I_{1}=\{3,4\}$, the strict augmentation property (IsA) implies that $\{2,3\}$ or $\{2,4\}$ should be an independent set. But neither $\{2,3\}$ nor $\{2,4\}$ is included in any member of $\mathcal{B}$. Hence the present cg-matroid does not satisfy the strict augmentation property (IsA).

Let $S_{1}=\{1,2,3,6,7\}$ and $S_{2}=\{2,3,5,7\}$. Then $S_{1}$ and $S_{2}$ are spanning sets of the cg-matroid. Since $\left|S_{1}\right|>\left|S_{2}\right|$ and $S_{1} \backslash S_{2}=\{1,6\}$, the strict reduction property (SsR) implies that $\{2,3,6,7\}$ or $\{1,2,3,7\}$ should be a spanning set. But we have $\{1,2,3,7\} \notin \mathcal{F}^{\prime}$, and $\{2,3,6,7\}$ does not contain any member of $\mathcal{B}$. Hence the present cg-matroid does not satisfy the strict reduction property (SsR).

Tame cg-matroids are characterized as follows:

Theorem 4.17. Let $M=(E, \mathcal{F} ; \mathcal{B})$ be a cg-matroid with a family $\mathcal{B}$ of bases. Then $M$ is a tame cg-matroid if and only if $\mathcal{B}$ satisfies the properties (BS) and $(\mathrm{BcS})$.

Proof. This follows from Theorems 4.6 and 4.12.

The relations between subclasses of cg-matroids are shown in Figure 5.

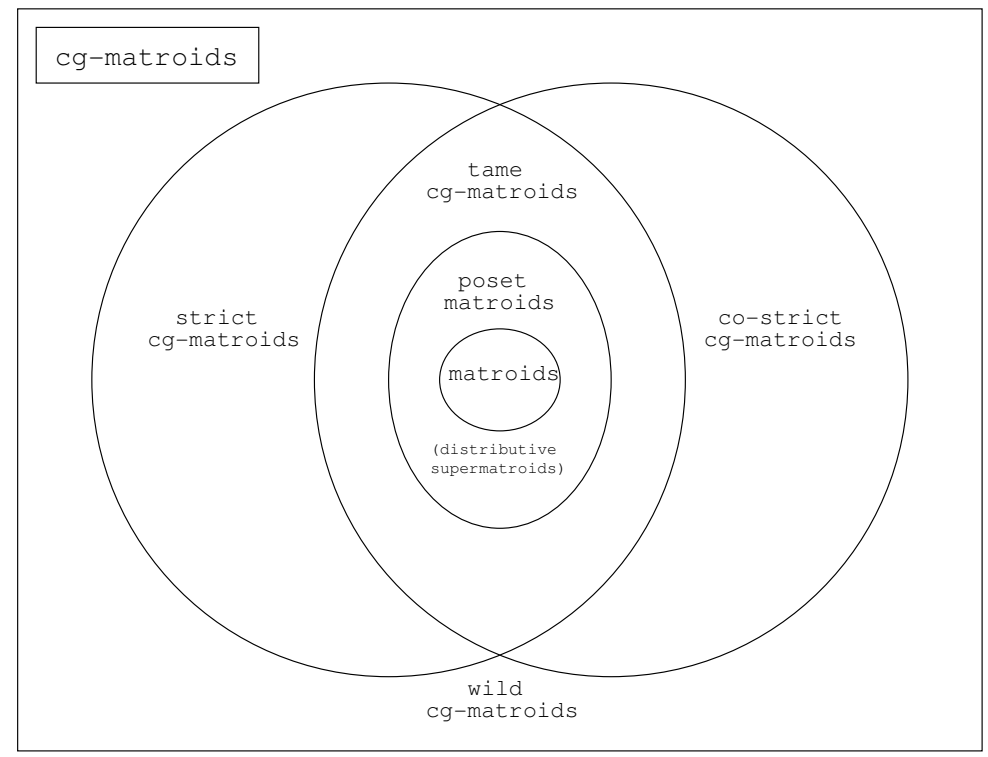

Figure 5. Subclasses of cg-matroids. 


\section{$\S 5$. Operations on cg-matroids}

In this section, we consider various operations on cg-matroids.

\section{§5.1. Restriction}

In this subsection, we discuss the restriction of cg-matroids.

Definition. Let $M=(E, \mathcal{F} ; \mathcal{I})$ be a cg-matroid with a family $\mathcal{I}$ of independent sets, and let $X$ be a closed set. The restriction

$$
M|X=(E, \mathcal{F} ; \mathcal{I})| X:=\left(X, \mathcal{F}^{(X)} ; \mathcal{I}^{(X)}\right)
$$

of the cg-matroid $M$ to the closed set $X$ is the pair of the restriction $\left(X, \mathcal{F}^{(X)}\right)$ of the convex geometry $(E, \mathcal{F})$ to $X$ and the restriction $\mathcal{I}^{(X)}$ of the family $\mathcal{I}$ of independent sets to $X$.

Lemma 5.1. Let $M=(E, \mathcal{F} ; \mathcal{I})$ be a cg-matroid and $X$ a closed set. The restriction $M \mid X=\left(X, \mathcal{F}^{(X)} ; \mathcal{I}^{(X)}\right)$ of $M$ to $X$ satisfies the properties (I0) and (I1).

Proof. Since $\emptyset \in \mathcal{I}$, we have $\emptyset=X \cap \emptyset \in \mathcal{I}^{(X)}$, and thus $\mathcal{I}^{(X)}$ satisfies the property (I0). Take any $I_{1} \in \mathcal{F}^{(X)}$ and $I_{2} \in \mathcal{I}^{(X)}$ with $I_{1} \subseteq I_{2}$. Then $I_{1} \in \mathcal{F}, I_{1} \subseteq X$, and $I_{2} \in \mathcal{I}$. By the property (I1) for $\mathcal{I}$, we have $I_{1} \in \mathcal{I}$. Therefore we have $I_{1} \in \mathcal{I}^{(X)}$, and thus $\mathcal{I}^{(X)}$ satisfies the property (I1).

Lemma 5.2. Let $M=(E, \mathcal{F} ; \mathcal{I})$ be a cg-matroid and $S$ a spanning set of $M$. Then $\operatorname{Max}\left(\mathcal{I}^{(S)}\right) \subseteq \operatorname{Max}(\mathcal{I})$.

Proof. Take any $I \in \operatorname{Max}\left(\mathcal{I}^{(S)}\right)$. Then $I \subseteq S$ and $I \in \mathcal{I}$. By the middle base property $(\mathrm{BM})$, there exists $B \in \mathcal{B}=\operatorname{Max}(\mathcal{I})$ such that $I \subseteq B \subseteq S$. Since $I$ is maximal in $\mathcal{I}^{(S)}$, it follows that $I=B$. Thus we have $I \in \operatorname{Max}(\mathcal{I})$.

Theorem 5.3. Let $M=(E, \mathcal{F} ; \mathcal{I})$ be a cg-matroid and $S$ a spanning set of $M$. The restriction $M \mid S=\left(S, \mathcal{F}^{(S)} ; \mathcal{I}^{(S)}\right)$ of $M$ to $S$ is also a cg-matroid.

Proof. We show that $\mathcal{I}^{(S)}$ satisfies the property (IA). Take any $I_{1} \in \mathcal{I}^{(S)}$ and $I_{2} \in \operatorname{Max}\left(\mathcal{I}^{(S)}\right)$ with $\left|I_{1}\right|<\left|I_{2}\right|$. Then $I_{1} \in \mathcal{I}, I_{2} \in \operatorname{Max}(\mathcal{I})$ by Lemma 5.2, and $I_{1}, I_{2} \subseteq S$. By the property (IA) for $\mathcal{I}$, there exists $e \in \tau\left(I_{1} \cup I_{2}\right) \backslash I_{1}$ such that $I_{1} \cup\{e\} \in \mathcal{I}$, where $\tau$ is the closure operator associated with the convex geometry $(E, \mathcal{F})$. Since $I_{1}, I_{2} \subseteq S$, we have $I_{1} \cup I_{2} \subseteq S$. Then $\tau\left(I_{1} \cup I_{2}\right) \subseteq \tau(S)=S$ by (cl2) and $S \in \mathcal{F}$. Since $\tau\left(I_{1} \cup I_{2}\right) \subseteq S$, we have $I_{1} \cup\{e\} \subseteq S$. By the definition of the closure operator of a convex geometry and $I_{1} \cup I_{2} \subseteq S$, we have $\tau\left(I_{1} \cup I_{2}\right)=$ $\tau^{\prime}\left(I_{1} \cup I_{2}\right)$, where $\tau^{\prime}$ is the closure operator associated with the convex geometry 
$\left(S, \mathcal{F}^{(S)}\right)$. Therefore $I_{1} \cup\{e\} \in \mathcal{I}^{(S)}$ and $e \in \tau^{\prime}\left(I_{1} \cup I_{2}\right) \backslash I_{1}$, and thus $\mathcal{I}^{(S)}$ satisfies the property (IA). Hence $\left(S, \mathcal{F}^{(S)} ; \mathcal{I}^{(S)}\right)$ is a cg-matroid.

The following example shows that the restriction $M \mid X$ of a cg-matroid $M$ to a closed set $X$ which is not a spanning set of $M$ is not always a cg-matroid.

Example 5.4. Let $M=(E, \mathcal{F} ; \mathcal{B})$ be the (non-strict) cg-matroid given in Example 4.1. Take $X=\{1,4,5\}$, which is a closed set but not a spanning set of $M$. Then the restriction of $M$ to $X$ is $M \mid X=\left(X, \mathcal{F}^{(X)} ; \mathcal{I}^{(X)}\right)$, where $\mathcal{F}^{(X)}=\{\emptyset,\{1\}$, $\{4\},\{5\},\{1,4\},\{1,5\},\{4,5\},\{1,4,5\}\}$ and $\mathcal{I}^{(X)}=\{\emptyset,\{1\},\{4\},\{5\},\{4,5\}\}$ (see Figure 6). But $M \mid X$ is not a cg-matroid since the elements of $\mathcal{B}(M \mid X)=$ $\operatorname{Max}\left(\mathcal{I}^{(X)}\right)=\{\{1\},\{4,5\}\}$ do not have the same cardinality.

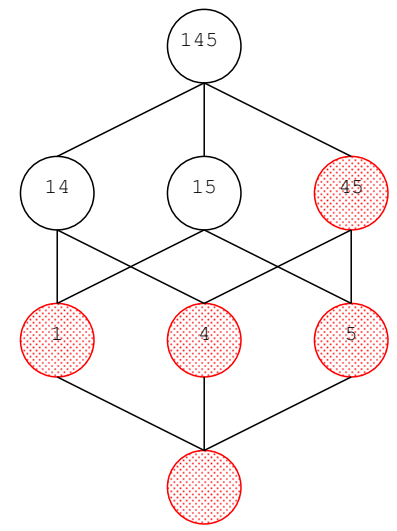

Figure 6. Restriction of a non-strict cg-matroid.

In the case of a strict cg-matroid, the restriction to a closed set is always a strict cg-matroid.

Theorem 5.5. Let $M=(E, \mathcal{F} ; \mathcal{I})$ be a strict cg-matroid and $X$ a closed set. The restriction $M \mid X=\left(X, \mathcal{F}^{(X)} ; \mathcal{I}^{(X)}\right)$ of $M$ to $X$ is also a strict cg-matroid.

Proof. We show that $\mathcal{I}^{(X)}$ satisfies the property (IsA). Take any $I_{1}, I_{2} \in \mathcal{I}^{(X)}$ with $\left|I_{1}\right|<\left|I_{2}\right|$. Then $I_{1}, I_{2} \in \mathcal{I}$ and $I_{1}, I_{2} \subseteq X$. By the property (IsA) for $\mathcal{I}$, there exists $e \in \tau\left(I_{1} \cup I_{2}\right) \backslash I_{1}$ such that $I_{1} \cup\{e\} \in \mathcal{I}$, where $\tau$ is the closure operator associated with the convex geometry $(E, \mathcal{F})$. Since $\tau\left(I_{1} \cup I_{2}\right) \subseteq X$, we have $I_{1} \cup\{e\} \subseteq X$. Note that $\tau\left(I_{1} \cup I_{2}\right)=\tau^{\prime}\left(I_{1} \cup I_{2}\right)$, where $\tau^{\prime}$ is the closure operator associated with the convex geometry $\left(X, \mathcal{F}^{(X)}\right)$. Therefore we have $I_{1} \cup\{e\} \in \mathcal{I}^{(X)}$ and $e \in \tau^{\prime}\left(I_{1} \cup I_{2}\right) \backslash I_{1}$, and thus $\mathcal{I}^{(X)}$ satisfies the property (IsA). Hence $\left(X, \mathcal{F}^{(X)} ; \mathcal{I}^{(X)}\right)$ is a strict cg-matroid. 
Proposition 5.6. Let $M=(E, \mathcal{F} ; \mathcal{B})$ be a cg-matroid and $X$ a closed set. If the restriction $M \mid X$ of $M$ to $X$ is a cg-matroid, then the family of bases of $M \mid X$ is given by

$$
\mathcal{B}(M \mid X)=\operatorname{Max}\{B \cap X \mid B \in \mathcal{B}\} .
$$

If in addition $X$ is a spanning set of $M$, then the family of bases of $M \mid X$ is also given by

$$
\mathcal{B}(M \mid X)=\operatorname{Max}\{B \in \mathcal{B} \mid B \subseteq X\} .
$$

Proof. The first statement follows since $\mathcal{B}(M \mid X)=\operatorname{Max}\left(\mathcal{I}^{(X)}\right)=\operatorname{Max}\{I \cap X \mid$ $I \in \mathcal{I}\}=\operatorname{Max}\{B \cap X \mid B \in \mathcal{B}\}$, where $\mathcal{I}=\mathcal{I}(M)$ is the family of independent sets of $M$. If $X$ is a spanning set of $M$, then we have $\operatorname{Max}\{B \cap X \mid B \in \mathcal{B}\}=$ $\operatorname{Max}\{B \in \mathcal{B} \mid B \subseteq X\}$, proving the second statement.

\section{$\S 5.2$. Contraction}

In this subsection, we discuss the contraction of cg-matroids.

Definition. Let $M=(E, \mathcal{F} ; \mathcal{S})$ be a cg-matroid with the family $\mathcal{S}$ of spanning sets, and let $X$ be a closed set. The contraction

$$
M / X=(E, \mathcal{F} ; \mathcal{S}) / X:=\left(E \backslash X, \mathcal{F}_{(X)} ; \mathcal{S}_{(X)}\right)
$$

of $M$ by $X$ is the pair of the contraction $\left(E \backslash X, \mathcal{F}_{(X)}\right)$ of the convex geometry $(E, \mathcal{F})$ by $X$ and the contraction $\mathcal{S}_{(X)}$ of the family $\mathcal{S}$ of spanning sets by $X$.

Lemma 5.7. Let $M=(E, \mathcal{F} ; \mathcal{S})$ be a cg-matroid and $X$ a closed set. The contraction $M / X=\left(E \backslash X, \mathcal{F}_{(X)} ; \mathcal{S}_{(X)}\right)$ of $M$ by $X$ satisfies the properties (S0) and (S1).

Proof. Since $E \in \mathcal{S}$ and $E \supseteq X$, we have $E \backslash X \in \mathcal{S}_{(X)}$, and thus $\mathcal{S}_{(X)}$ satisfies the property (S0). Take any $S_{1} \backslash X \in \mathcal{F}_{(X)}$ with $S_{1} \in \mathcal{F}, S_{1} \supseteq X$ and $S_{2} \backslash X \in \mathcal{S}_{(X)}$ with $S_{2} \in \mathcal{S}, S_{2} \supseteq X$ such that $S_{1} \backslash X \supseteq S_{2} \backslash X$. Then $S_{1} \supseteq S_{2}$. By the property (S1) for $\mathcal{S}$, we have $S_{1} \in \mathcal{S}$. Therefore we have $S_{1} \backslash X \in \mathcal{S}_{(X)}$, and thus $\mathcal{S}_{(X)}$ satisfies the property (S1).

Lemma 5.8. Let $M=(E, \mathcal{F} ; \mathcal{S})$ be a cg-matroid and $I$ an independent set of $M$. Then $\operatorname{Min}\left(\mathcal{S}_{[I]}\right) \subseteq \operatorname{Min}(\mathcal{S})$.

Proof. Take any $S \in \operatorname{Min}\left(\mathcal{S}_{[I]}\right)$. Then $I \subseteq S$ and $S \in \mathcal{S}$. By the middle base property $(\mathrm{BM})$, there exists $B \in \mathcal{B}=\operatorname{Min}(\mathcal{S})$ such that $I \subseteq B \subseteq S$. Since $S$ is minimal in $\mathcal{S}_{[I]}$, it follows that $S=B$. Thus we have $S \in \operatorname{Min}(\mathcal{S})$. 
Lemma 5.9. Let $M=(E, \mathcal{F} ; \mathcal{S})$ be a cg-matroid and $I$ an independent set of $M$. For any $T \in \operatorname{Min}\left(\mathcal{S}_{(I)}\right)$, there exists $S \in \operatorname{Min}(\mathcal{S})$ such that $T=S \backslash I$.

Proof. This follows from the definition of contraction and Lemma 5.8.

Theorem 5.10. Let $M=(E, \mathcal{F} ; \mathcal{S})$ be a cg-matroid and $I$ an independent set of $M$. The contraction $M / I=\left(E \backslash I, \mathcal{F}_{(I)} ; \mathcal{S}_{(I)}\right)$ of $M$ by $I$ is also a cg-matroid.

Proof. We show that $\mathcal{S}_{(I)}$ satisfies the property (SR). Take any $S_{1} \backslash I \in \mathcal{S}_{(I)}$ and $S_{2} \backslash I \in \operatorname{Min}\left(\mathcal{S}_{(I)}\right)$ such that $\left|S_{1} \backslash I\right|>\left|S_{2} \backslash I\right|$. Here we may assume that $I \subseteq S_{1} \in \mathcal{S}$ and $I \subseteq S_{2} \in \operatorname{Min}(\mathcal{S})$ by Lemma 5.9. Then $\left|S_{1}\right|>\left|S_{2}\right|$. By the property (SR) for $\mathcal{S}$, there exists $e \in S_{1} \backslash S_{2}$ such that $S_{1} \backslash\{e\} \in \mathcal{S}$. Since $e \notin S_{2} \supseteq I$, we have $S_{1} \backslash\{e\} \supseteq I$. Therefore we have $\left(S_{1} \backslash\{e\}\right) \backslash I \in \mathcal{S}_{(I)}$ and $e \in\left(S_{1} \backslash I\right) \backslash\left(S_{2} \backslash I\right)$, and thus $\mathcal{S}_{(I)}$ satisfies the property $(\mathrm{SR})$. By Lemma 5.7, $\left(E \backslash I, \mathcal{F}_{(I)} ; \mathcal{S}_{(I)}\right)$ is a cg-matroid.

The following example shows that the contraction $M / X$ of a cg-matroid $M$ by a closed set $X$ which is not an independent set of $M$ is not always a cg-matroid.

Example 5.11. Let $M=(E, \mathcal{F} ; \mathcal{B})$ be the (non-co-strict) cg-matroid given in Example 4.7. Take $X=\{1,3\} \in \mathcal{F}$ which is a closed set but not an independent set of $M$. Then the contraction of $M$ by $X$ is $M / X=\left(E \backslash X, \mathcal{F}_{(X)} ; \mathcal{S}_{(X)}\right)$, where $\mathcal{F}_{(X)}=\{\emptyset,\{2\},\{5\},\{2,4\},\{2,5\},\{2,4,5\}\}$ and $\mathcal{S}_{(X)}=\{\{5\},\{2,4\}$, $\{2,5\},\{2,4,5\}\}$ (see Figure 7 ). But $M / X$ is not a cg-matroid since the elements of $\mathcal{B}(M / X)=\operatorname{Min}\left(\mathcal{S}_{(X)}\right)=\{\{2,4\},\{5\}\}$ do not have the same cardinality.

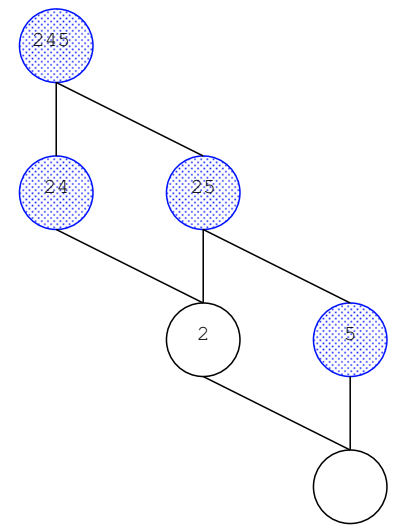

Figure 7. Contraction of a non-co-strict cg-matroid.

In the case of a co-strict cg-matroid, the contraction by a closed set is always a co-strict cg-matroid. 
Theorem 5.12. Let $M=(E, \mathcal{F} ; \mathcal{S})$ be a co-strict cg-matroid and $X$ a closed set. The contraction $M / X=\left(E \backslash X, \mathcal{F}_{(X)} ; \mathcal{S}_{(X)}\right)$ of $M$ by $X$ is also a co-strict cg-matroid.

Proof. Take any $S_{1} \backslash X, S_{2} \backslash X \in \mathcal{S}_{(X)}$ with $S_{1}, S_{2} \in \mathcal{S}, S_{1} \supseteq X, S_{2} \supseteq X$ such that $\left|S_{1} \backslash X\right|>\left|S_{2} \backslash X\right|$. Then $\left|S_{1}\right|>\left|S_{2}\right|$. By the property (SsR) for $\mathcal{S}$, there exists $e \in S_{1} \backslash S_{2}$ such that $S_{1} \backslash\{e\} \in \mathcal{S}$. Since $e \notin S_{2} \supseteq X$, we have $S_{1} \backslash\{e\} \supseteq X$. Therefore $\left(S_{1} \backslash\{e\}\right) \backslash X \in \mathcal{S}_{(X)}$ and $e \in\left(S_{1} \backslash X\right) \backslash\left(S_{2} \backslash X\right)$, and thus $\mathcal{S}_{(X)}$ satisfies the property $(\mathrm{SsR})$. By Lemma $5.7,\left(E \backslash X, \mathcal{F}_{(X)} ; \mathcal{S}_{(X)}\right)$ is a co-strict cg-matroid.

Proposition 5.13. Let $M=(E, \mathcal{F} ; \mathcal{B})$ be a cg-matroid and $X$ a closed set. If the contraction $M / X$ of $M$ by $X$ is a cg-matroid, then the family of bases of $M / X$ is given by

$$
\mathcal{B}(M / X)=\operatorname{Min}\{\tau(B \cup X) \backslash X \mid B \in \mathcal{B}\}
$$

If in addition $X$ is an independent set of $M$, then the family of bases of $M / X$ is also given by

$$
\mathcal{B}(M / X)=\operatorname{Min}\{B \backslash X \mid B \in \mathcal{B}, B \supseteq X\} .
$$

Proof. The first statement follows since $\mathcal{B}(M / X)=\operatorname{Min}\left(\mathcal{S}_{(X)}\right)=\operatorname{Min}\{\tau(S \cup X) \backslash$ $X \mid S \in \mathcal{S}\}=\operatorname{Min}\{\tau(B \cup X) \backslash X \mid B \in \mathcal{B}\}$, where $\mathcal{S}=\mathcal{S}(M)$ is the family of spanning sets of $M$. If $X$ is an independent set of $M$, then $\operatorname{Min}\{\tau(B \cup X) \backslash X \mid$ $B \in \mathcal{B}\}=\operatorname{Min}\{B \in \mathcal{B} \mid B \supseteq X\}$, proving the second statement.

\section{§5.3. Other operations}

Definition. A system obtained by restriction and contraction of a cg-matroid is called a minor of the cg-matroid.

Proposition 5.14. A minor of a tame cg-matroid is also a tame cg-matroid.

Proof. This follows from Theorems 5.5 and 5.12.

Next, we define truncation and elongation for cg-matroids.

Definition. Let $M=(E, \mathcal{F} ; \mathcal{I})$ be a cg-matroid with a family $\mathcal{I}$ of independent sets and $k$ an integer such that $0 \leq k \leq|E|$. The $k$-truncation $M^{k}=\left(E, \mathcal{F} ; \mathcal{I}^{k}\right)$ of $M$ is the pair of the convex geometry $(E, \mathcal{F})$ and $\mathcal{I}^{k}:=\{I \in \mathcal{I}|| I \mid \leq k\}$.

Theorem 5.15. Let $M=(E, \mathcal{F} ; \mathcal{I})$ be a strict cg-matroid and $k$ an integer such that $0 \leq k \leq|E|$. Then the $k$-truncation $M^{k}=\left(E, \mathcal{F} ; \mathcal{I}^{k}\right)$ of $M$ is also a strict cg-matroid. 
Proof. This follows from the fact that the properties (I0), (I1), and (IsA) for $\mathcal{I}$ imply the same properties for $\mathcal{I}^{k}$.

Definition. Let $M=(E, \mathcal{F} ; \mathcal{S})$ be a cg-matroid with a family $\mathcal{S}$ of spanning sets and $k$ an integer such that $0 \leq k \leq|E|$. The $k$-elongation $M_{k}=\left(E, \mathcal{F} ; \mathcal{S}_{k}\right)$ of $M$ is the pair of the convex geometry $(E, \mathcal{F})$ and $\mathcal{S}_{k}:=\{S \in \mathcal{S}|| S \mid \geq k\}$.

Theorem 5.16. Let $M=(E, \mathcal{F} ; \mathcal{S})$ be a co-strict cg-matroid and $k$ an integer such that $0 \leq k \leq|E|$. Then the $k$-elongation $M_{k}=\left(E, \mathcal{F} ; \mathcal{S}_{k}\right)$ of $M$ is also a co-strict cg-matroid.

Proof. This follows from the fact that the properties $(\mathrm{S} 0),(\mathrm{S} 1)$, and $(\mathrm{SsR})$ for $\mathcal{S}$ imply the same properties for $\mathcal{S}_{k}$.

We define the union of two cg-matroids.

Definition. Let $M_{1}=\left(E_{1}, \mathcal{F}_{1} ; \mathcal{B}_{1}\right)$ and $M_{2}=\left(E_{2}, \mathcal{F}_{2} ; \mathcal{B}_{2}\right)$ be cg-matroids with $E_{1} \cap E_{2}=\emptyset$. The union

$$
M_{1} \oplus M_{2}:=\left(E_{1} \cup E_{2}, \mathcal{F}_{1} \sqcup \mathcal{F}_{2} ; \mathcal{B}_{1} \sqcup \mathcal{B}_{2}\right)
$$

of the cg-matroids $M_{1}$ and $M_{2}$ is the pair of the union $\left(E_{1} \cup E_{2}, \mathcal{F}_{1} \sqcup \mathcal{F}_{2}\right)$ of the convex geometries $\left(E_{1}, \mathcal{F}_{1}\right)$ and $\left(E_{2} ; \mathcal{F}_{2}\right)$ and the union

$$
\mathcal{B}_{1} \sqcup \mathcal{B}_{2}:=\left\{B_{1} \cup B_{2} \mid B_{1} \in \mathcal{B}_{1}, B_{2} \in \mathcal{B}_{2}\right\}
$$

of $\mathcal{B}_{1}$ and $\mathcal{B}_{2}$.

Theorem 5.17. Let $M_{1}=\left(E_{1}, \mathcal{F}_{1} ; \mathcal{B}_{1}\right)$ and $M_{2}=\left(E_{2}, \mathcal{F}_{2} ; \mathcal{B}_{2}\right)$ be cg-matroids with $E_{1} \cap E_{2}=\emptyset$. Then $M_{1} \oplus M_{2}$ is a cg-matroid.

Proof. By the property $(\mathrm{B} 0)$ for $\mathcal{B}_{1}$ and $\mathcal{B}_{2}$, we have $\mathcal{B}_{1} \neq \emptyset$ and $\mathcal{B}_{2} \neq \emptyset$. Therefore we have $\mathcal{B}_{1} \sqcup \mathcal{B}_{2} \neq \emptyset$, and thus $\mathcal{B}_{1} \sqcup \mathcal{B}_{2}$ satisfies the property (B0).

Let $B_{1} \cup B_{2}, B_{1}^{\prime} \cup B_{2}^{\prime} \in \mathcal{B}_{1} \sqcup \mathcal{B}_{2}$ with $B_{1} \cup B_{2} \subseteq B_{1}^{\prime} \cup B_{2}^{\prime}$. Then it follows that $B_{1} \subseteq B_{1}^{\prime}$ and $B_{2} \subseteq B_{2}^{\prime}$ since $E_{1} \cap E_{2}=\emptyset$. By the property (B1) for $\mathcal{B}_{1}$ and $\mathcal{B}_{2}$, we have $B_{1}=B_{1}^{\prime}$ and $B_{2}=B_{2}^{\prime}$. Therefore we have $B_{1} \cup B_{2}=B_{1}^{\prime} \cup B_{2}^{\prime}$, and thus $\mathcal{B}_{1} \sqcup \mathcal{B}_{2}$ satisfies the property (B1).

Take $X_{1} \cup X_{2}, Y_{1} \cup Y_{2} \in \mathcal{F}_{1} \sqcup \mathcal{F}_{2}$ and $B_{1}^{\prime} \cup B_{2}^{\prime}, B_{1}^{\prime \prime} \cup B_{2}^{\prime \prime} \in \mathcal{B}_{1} \sqcup \mathcal{B}_{2}$ such that $B_{1}^{\prime} \cup B_{2}^{\prime} \supseteq X_{1} \cup X_{2} \subseteq Y_{1} \cup Y_{2} \supseteq B_{1}^{\prime \prime} \cup B_{2}^{\prime \prime}$. Since $E_{1} \cap E_{2}=\emptyset$, we have $B_{1}^{\prime} \supseteq X_{1} \subseteq Y_{1} \supseteq B_{1}^{\prime \prime}$ and $B_{2}^{\prime} \supseteq X_{2} \subseteq Y_{2} \supseteq B_{2}^{\prime \prime}$. By the middle base property (BM) for $\mathcal{B}_{1}$ and $\mathcal{B}_{2}$, there exists $B_{1} \in \mathcal{B}_{1}$ such that $X_{1} \subseteq B_{1} \subseteq Y_{1}$ and there exists $B_{2} \in \mathcal{B}_{2}$ such that $X_{2} \subseteq B_{2} \subseteq Y_{2}$. Then we have $B_{1} \cup B_{2} \in \mathcal{B}_{1} \sqcup \mathcal{B}_{2}$ and $X_{1} \cup X_{2} \subseteq$ $B_{1} \cup B_{2} \subseteq Y_{1} \cup Y_{2}$, and thus $\mathcal{B}_{1} \sqcup \mathcal{B}_{2}$ satisfies the middle base property (BM).

Hence $M_{1} \oplus M_{2}$ is a cg-matroid. 


\section{§6. Optimization on cg-matroids}

In this section, we consider an optimization problem on cg-matroids, which reveals the relation between the greedy algorithm and cg-matroids.

\section{§6.1. Maximum base problem and the greedy algorithm}

In this subsection, we consider the maximum base problem of a cg-matroid.

Let $M=(E, \mathcal{F} ; \mathcal{B})$ be a cg-matroid with a family $\mathcal{B}$ of bases, and $w: E \rightarrow \mathbb{R}_{\geq 0}$ be a nonnegative weight function on $E$. We denote $\sum_{e \in X} w(e)$ by $w(X)$.

The maximum base problem is the following:

$$
\begin{aligned}
P_{\max }(\mathcal{B}, w): & \text { maximize } w(B) \\
& \text { subject to } B \in \mathcal{B}(M)
\end{aligned}
$$

Since the family $\mathcal{B}(M)$ of bases is the family of maximal elements in the family $\mathcal{I}(M)$ of independent sets and the weights are nonnegative, the above maximum base problem has the same optimal solution as the following maximum independent set problem.

Let $M=(E, \mathcal{F} ; \mathcal{I})$ be a cg-matroid with the family $\mathcal{I}$ of independent sets, and $w: E \rightarrow \mathbb{R}_{\geq 0}$ be a nonnegative weight function on $E$. The maximum independent set problem is the following:

$$
\begin{aligned}
P_{\max }(\mathcal{I}, w): & \text { maximize } w(I) \\
& \text { subject to } I \in \mathcal{I}(M)
\end{aligned}
$$

In the following, we consider this problem, more generally, for a hereditary system on a convex geometry which is defined as follows.

Definition. Let $(E, \mathcal{F})$ be a convex geometry and $\mathcal{I}$ be a subfamily of $\mathcal{F}$. We call the pair $(E, \mathcal{F} ; \mathcal{I})$ a hereditary system on a convex geometry or a $c g$-independence system if $\mathcal{I}$ satisfies the properties (I0) and (I1).

Definition. Let $(E, \mathcal{F})$ be a convex geometry and $X$ be a closed set, where $1 \leq$ $|X|=k \leq|E|$. An ordering $\left(e_{1}, \ldots, e_{k}\right)$ of the elements of $X$ is called $\mathcal{F}$-feasible if $X_{i}:=\left\{e_{1}, \ldots, e_{i}\right\} \in \mathcal{F}$ for all $1 \leq i \leq k$.

Definition. Let $(E, \mathcal{F})$ be a convex geometry and $w: E \rightarrow \mathbb{R}_{\geq 0}$ be a nonnegative weight function on $E$. Then $w$ is called a natural weighting on $(E, \mathcal{F})$ if there exists an $\mathcal{F}$-feasible ordering $\left(e_{1}, \ldots, e_{n}\right)$ of $E$ such that $w\left(e_{1}\right) \geq \cdots \geq w\left(e_{n}\right)$.

Lemma 6.1. Let $(E, \mathcal{F})$ be a convex geometry and $w: E \rightarrow \mathbb{R}_{\geq 0}$ be a natural weighting on $(E, \mathcal{F})$. Then, for any closed set $X$, there exists an $\mathcal{F}$-feasible ordering $\left(e_{1}, \ldots, e_{k}\right)$ of $X$ such that $w\left(e_{1}\right) \geq \cdots \geq w\left(e_{k}\right)$. 
Proof. Since $w: E \rightarrow \mathbb{R}_{\geq 0}$ is a natural weighting on $(E, \mathcal{F})$, there exists an $\mathcal{F}$-feasible ordering $\left(e_{1}, \ldots, e_{n}\right)$ of $E$ such that $w\left(e_{1}\right) \geq \cdots \geq w\left(e_{n}\right)$. Put $Y_{i}=$ $\left\{e_{1}, \ldots, e_{i}\right\} \in \mathcal{F}(1 \leq i \leq n)$ and $Y_{0}=\emptyset$. Also put $Z_{i}=X \cap Y_{i}(0 \leq i \leq n)$. Then we have $Z_{i} \in \mathcal{F}$ by the property (CG1) and

$$
\emptyset=Z_{0} \subseteq Z_{1} \subseteq \cdots \subseteq Z_{n}=X .
$$

We can take the strictly increasing maximal subchain of this chain,

$$
\emptyset=Z_{i_{0}} \subsetneq Z_{i_{1}} \subsetneq \cdots \subsetneq Z_{i_{k}}=X,
$$

where $k=|X|$. Take $\hat{e}_{t} \in Z_{i_{t}} \backslash Z_{i_{t-1}}(1 \leq t \leq k)$. Then $\left(\hat{e}_{1}, \ldots, \hat{e}_{k}\right)$ is an $\mathcal{F}$-feasible ordering of $X$ such that $w\left(\hat{e}_{1}\right) \geq \cdots \geq w\left(\hat{e}_{k}\right)$. Thus the lemma follows.

Lemma 6.2. Let $(E, \mathcal{F})$ be a convex geometry and $w: E \rightarrow \mathbb{R}_{\geq 0}$ be a natural weighting of $(E, \mathcal{F})$. Then, for any closed set $X$, there exists $\hat{e} \in \operatorname{ex}(X)$ such that $w(\hat{e})=\min \{w(e) \mid e \in X\}$.

Proof. Take a closed set $X$. Then, from Lemma 6.1, there exists an $\mathcal{F}$-feasible ordering $\left(e_{1}, \ldots, e_{k}\right)$ of $X$ such that $w\left(e_{1}\right) \geq \cdots \geq w\left(e_{k}\right)$, where $k=|X|$. Since $\left\{e_{1}, \ldots, e_{k-1}\right\} \in \mathcal{F}$, we have $\hat{e}:=e_{k} \in \operatorname{ex}(X)$ and $w(\hat{e})=\min \{w(e) \mid e \in X\}$.

The greedy algorithm (or the best-in greedy algorithm) is the following:

\section{Greedy Algorithm (Best-In Greedy Algorithm).}

- Initialization: Set $I^{(0)} \leftarrow \emptyset$.

- Iteration: For $i=0$ to $n-1$, do the following:

step $i$ : If there exists $e \in E \backslash I^{(i)}$ such that $I^{(i)} \cup\{e\} \in \mathcal{I}$, then choose such an element $e_{i+1}$ of maximum weight, i.e.,

$$
w\left(e_{i+1}\right)=\max \left\{w(e) \mid e \in E \backslash I^{(i)}, I^{(i)} \cup\{e\} \in \mathcal{I}\right\} .
$$

Let $I^{(i+1)} \leftarrow I^{(i)} \cup\left\{e_{i+1}\right\}$ and go to step $i+1$.

Otherwise, let $I_{\mathrm{GA}} \leftarrow I^{(i)}$ and go to Termination step.

- Termination: Output $I_{\mathrm{GA}}$.

\section{$\S 6.2$. Characterization}

Now, we show that the greedy algorithm works for a hereditary system on a convex geometry with any natural weighting if and only if the hereditary system is a strict cg-matroid.

First, we show that the greedy algorithm works for any strict cg-matroid with any natural weighting. 
Theorem 6.3. Let $(E, \mathcal{F} ; \mathcal{I})$ be a strict cg-matroid. Then the greedy algorithm produces an optimal solution of $P_{\max }(\mathcal{I}, w)$ for $(E, \mathcal{F} ; \mathcal{I})$ with any natural weighting $w$ on $(E, \mathcal{F})$.

Proof. Fix any natural weighting $w: E \rightarrow \mathbb{R}_{\geq 0}$ on $(E, \mathcal{F})$. Let $I_{\mathrm{GA}}=\left\{e_{1}, \ldots, e_{r}\right\}$ $\in \mathcal{I}$ be a solution obtained by the greedy algorithm. Note that $\left(e_{1}, \ldots, e_{r}\right)$ is an $\mathcal{F}$ feasible ordering such that $w\left(e_{1}\right) \geq \cdots \geq w\left(e_{r}\right)$. Since $w$ is nonnegative, if $X \subseteq Y$ then $w(X) \leq w(Y)$. Take any $I^{\prime} \in \mathcal{I}$ which is maximal in $\mathcal{I}$. Then, from the property (IS), $I^{\prime}$ also has $r$ elements. From Lemma 6.1, there exists an $\mathcal{F}$-feasible ordering $\left(e_{1}^{\prime}, \ldots, e_{r}^{\prime}\right)$ of $I^{\prime}$ such that $w\left(e_{1}^{\prime}\right) \geq \cdots \geq w\left(e_{r}^{\prime}\right)$. Then it follows from Lemma 6.4 below that $w\left(e_{i}\right) \geq w\left(e_{i}^{\prime}\right)$ for all $1 \leq i \leq r$. Thus we have

$$
w\left(I_{\mathrm{GA}}\right)=\sum_{i=1}^{r} w\left(e_{i}\right) \geq \sum_{i=1}^{r} w\left(e_{i}^{\prime}\right)=w\left(I^{\prime}\right) .
$$

Hence $I_{\mathrm{GA}}$ is an optimal solution of the problem $P_{\max }(\mathcal{I}, w)$, and the theorem follows.

Lemma 6.4. In the setting of the proof of Theorem 6.3, we have $w\left(e_{i}\right) \geq w\left(e_{i}^{\prime}\right)$ for all $1 \leq i \leq r$.

Proof. Suppose that the conclusion does not hold. Let $k$ be the minimum number such that $w\left(e_{k}\right)<w\left(e_{k}^{\prime}\right)$. Put $I_{1}=\left\{e_{1}, \ldots, e_{k-1}\right\}$ and $I_{2}=\left\{e_{1}^{\prime}, \ldots, e_{k}^{\prime}\right\}$. Then we have $I_{1} \in \mathcal{F}$ and $I_{2} \in \mathcal{F}$ since $\left(e_{1}, \ldots, e_{r}\right)$ and $\left(e_{1}^{\prime}, \ldots, e_{r}^{\prime}\right)$ are $\mathcal{F}$-feasible orderings. Thus it follows from (I1) that $I_{1} \in \mathcal{I}$ and $I_{2} \in \mathcal{I}$. Since $\left|I_{1}\right|<\left|I_{2}\right|$, from (IsA), there exists $e^{\prime} \in \tau\left(I_{1} \cup I_{2}\right) \backslash I_{1}$ such that $I_{1} \cup\left\{e^{\prime}\right\} \in \mathcal{I}$. Here we have the following two cases.

Case 1: $e^{\prime} \in I_{2} \backslash I_{1}$.

Since $e_{k}^{\prime}$ has the minimum weight in $I_{2}$, we have $w\left(e^{\prime}\right) \geq w\left(e_{k}^{\prime}\right)>w\left(e_{k}\right)$. This is a contradiction to the choice of $e_{k}$ in step $k-1$ of the greedy algorithm.

Case 2: $e^{\prime} \in \tau\left(I_{1} \cup I_{2}\right) \backslash\left(I_{1} \cup I_{2}\right)$.

From Lemma 6.2, there exists $\hat{e} \in \operatorname{ex}\left(\tau\left(I_{1} \cup I_{2}\right)\right)$ such that $w(\hat{e})=\min \{w(e) \mid$ $\left.e \in \tau\left(I_{1} \cup I_{2}\right)\right\}$. Here, note that $\operatorname{ex}\left(\tau\left(I_{1} \cup I_{2}\right)\right) \subseteq I_{1} \cup I_{2}$ (cf. [9, (2.9)]). So we have $\hat{e} \in I_{1} \cup I_{2}$, and thus $e^{\prime} \neq \hat{e}$. Since $e_{k}^{\prime}$ has the minimum weight in $I_{1} \cup I_{2}$ and $\hat{e} \in I_{1} \cup I_{2}$, we have $w(\hat{e}) \geq w\left(e_{k}^{\prime}\right)$. Therefore

$$
w\left(e^{\prime}\right) \geq \min \left\{w(e) \mid e \in \tau\left(I_{1} \cup I_{2}\right)\right\}=w(\hat{e}) \geq w\left(e_{k}^{\prime}\right)>w\left(e_{k}\right) .
$$

This is a contradiction to the choice of $e_{k}$ in step $k-1$ of the greedy algorithm.

Hence the lemma follows. 
Next, we show that a hereditary system on a convex geometry for which the greedy algorithm works for any natural weighting is a strict cg-matroid.

Theorem 6.5. Let $(E, \mathcal{F} ; \mathcal{I})$ be a hereditary system on a convex geometry. Suppose that it satisfies the following property:

(IG) The greedy algorithm produces an optimal solution of $P_{\max }(\mathcal{I}, w)$ for $(E, \mathcal{F} ; \mathcal{I})$ with any natural weighting $w$ on $(E, \mathcal{F})$.

Then $(E, \mathcal{F} ; \mathcal{I})$ is a strict cg-matroid.

Proof. We will show that (IsA) holds. Take any $I_{1}, I_{2} \in \mathcal{I}$ such that $\left|I_{1}\right|<\left|I_{2}\right|$. If $I_{1} \subseteq I_{2}$ then it is easy to see that (IsA) holds. So we suppose that $I_{1} \nsubseteq I_{2}$, and suppose that (IsA) does not hold, i.e., there is no element $e \in \tau\left(I_{1} \cup I_{2}\right) \backslash I_{1}$ such that $I_{1} \cup\{e\} \in \mathcal{I}$.

Then we have $0<\left|I_{1}-I_{2}\right|=\left|I_{1}\right|-\left|I_{1} \cap I_{2}\right|<\left|I_{2}\right|-\left|I_{1} \cap I_{2}\right|=\left|I_{2}-I_{1}\right|$. Take a positive number $\varepsilon$ which satisfies $0<(1+\varepsilon)\left|I_{1}-I_{2}\right|<\left|I_{2}-I_{1}\right|$. Define a weight function $w: E \rightarrow \mathbb{R}_{\geq 0}$ as follows:

$$
w(e)= \begin{cases}2 & \left(e \in I_{1} \cap I_{2}\right), \\ 1 /\left|I_{1}-I_{2}\right| & \left(e \in I_{1} \backslash I_{2}\right), \\ (1+\varepsilon) /\left|I_{2}-I_{1}\right| & \left(e \in \tau\left(I_{1} \cup I_{2}\right) \backslash I_{1}\right), \\ 0 & \left(e \in E \backslash \tau\left(I_{1} \cup I_{2}\right)\right) .\end{cases}
$$

Then $w$ is a natural weighting on $(E, \mathcal{F})$, because any maximal chain of $\mathcal{F}$ that contains $I_{1} \cap I_{2}, I_{1}$, and $\tau\left(I_{1} \cup I_{2}\right)$ naturally defines an $\mathcal{F}$-feasible ordering $\left(e_{1}, \ldots, e_{n}\right)$ of $E$ such that $w\left(e_{1}\right) \geq \cdots \geq w\left(e_{n}\right)$.

Put $k=\left|I_{1}\right|$ and consider the greedy algorithm. In step $k-1$, we have $I^{(k)}=$ $I_{1}$. From the assumption, we cannot take an element $e \in \tau\left(I_{1} \cup I_{2}\right) \backslash I_{1}$ in step $k$. Let $I_{\mathrm{GA}} \in \mathcal{I}$ be a solution obtained by the greedy algorithm. We claim that $I_{\mathrm{GA}}$ does not contain any elements in $\tau\left(I_{1} \cup I_{2}\right) \backslash I_{1}$, i.e., $I_{\mathrm{GA}} \cap \tau\left(I_{1} \cup I_{2}\right)=I_{1}$. If there exist such elements $e_{i_{1}}, \ldots, e_{i_{t}}$, then consider a maximal chain in $\mathcal{F}$ which contains $I_{1}$ and the subset $I_{1} \cup\left\{e_{i_{1}}, \ldots, e_{i_{t}}\right\}=I_{\mathrm{GA}} \cap \tau\left(I_{1} \cup I_{2}\right) \in \mathcal{F}$. Then $I_{1} \cup\left\{e_{i}\right\} \in \mathcal{F}$ for some $e_{i} \in\left\{e_{i_{1}}, \ldots, e_{i_{t}}\right\}$. Since $I_{1} \cup\left\{e_{i}\right\} \subseteq I_{\mathrm{GA}} \in \mathcal{I}$, from (I1), we have $I_{1} \cup\left\{e_{i}\right\} \in \mathcal{I}$, but this is a contradiction to the assumption.

Now we have the following:

$$
\begin{aligned}
w\left(I_{\mathrm{GA}}\right) & =w\left(I_{1}\right)=2\left|I_{1} \cap I_{2}\right|+1, \\
w\left(I_{2}\right) & =2\left|I_{1} \cap I_{2}\right|+1+\varepsilon .
\end{aligned}
$$

Thus we have $w\left(I_{\mathrm{GA}}\right)<w\left(I_{2}\right)$, i.e., $I_{\mathrm{GA}}$ is not an optimal solution of $P_{\max }(\mathcal{I}, w)$. This is a contradiction to (IG).

Hence (IsA) holds, and thus $(E, \mathcal{F} ; \mathcal{I})$ is a strict cg-matroid. 
Combining Theorems 6.3 and 6.5, we get the following.

Theorem 6.6. Let $(E, \mathcal{F} ; \mathcal{I})$ be a hereditary system on a convex geometry. Then $(E, \mathcal{F} ; \mathcal{I})$ is a strict cg-matroid if and only if the greedy algorithm produces an optimal solution of $P_{\max }(\mathcal{I}, w)$ for $(E, \mathcal{F} ; \mathcal{I})$ with any natural weighting $w$ on $(E, \mathcal{F})$.

To end this subsection, we give some examples which show that the greedy algorithm fails for a strict cg-matroid with a non-natural weighting and also fails for a non-strict cg-matroid with a natural weighting.

Example 6.7. Let $(E, \mathcal{F})$ be the convex shelling of five points in $\mathbb{R}^{1}$, i.e., $E=$ $\{1,2,3,4,5\}$ and $\mathcal{F}=\{\emptyset,\{1\},\{2\},\{3\},\{4\},\{5\},\{1,2\},\{2,3\},\{3,4\},\{4,5\},\{1,2,3\}$, $\{2,3,4\},\{3,4,5\},\{1,2,3,4\},\{2,3,4,5\},\{1,2,3,4,5\}\}$. Consider the 3-uniform cg-matroid on this convex geometry $(E, \mathcal{F})$, i.e., $\mathcal{I}=\{X \in \mathcal{F}|| X \mid \leq 3\}$ (see Figure 8$)$. Then $(E, \mathcal{F} ; \mathcal{I})$ is a strict cg-matroid.
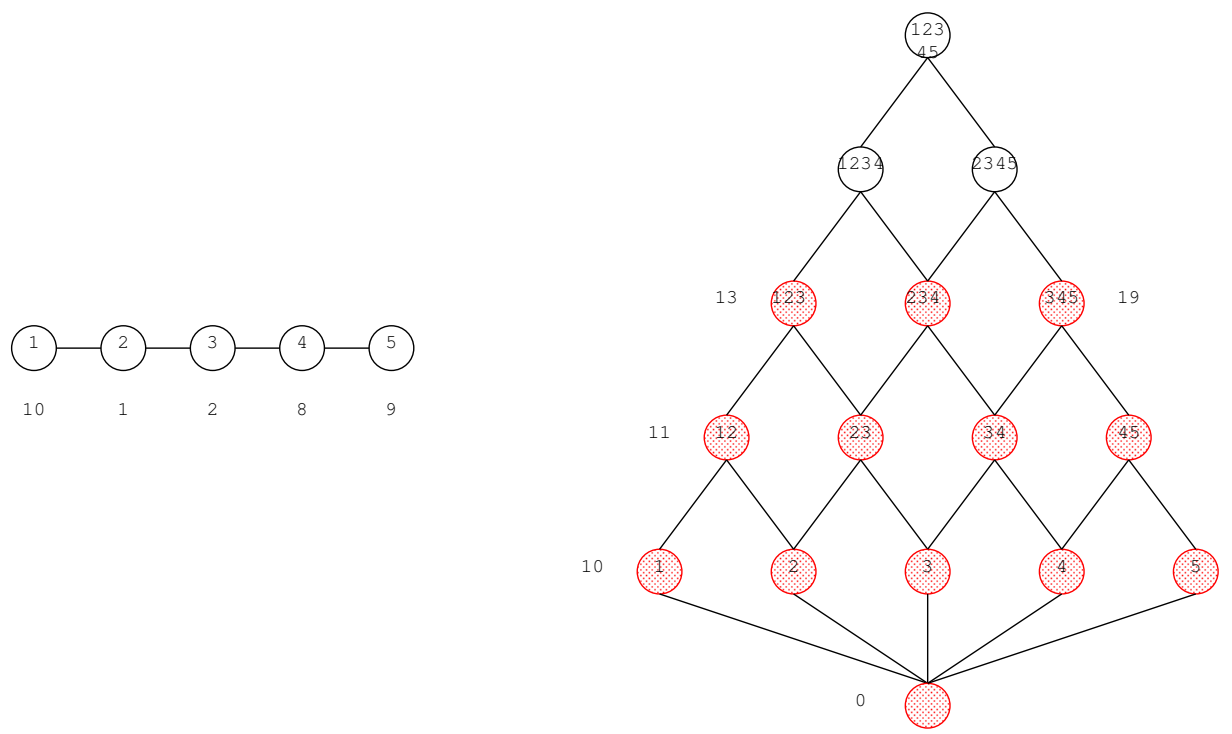

Figure 8. A tree shelling of a path with five vertices.

Let $w: E \rightarrow \mathbb{R}_{\geq 0}$ be a weight function on $E$ defined by $w(1)=10, w(2)=1$, $w(3)=2, w(4)=8, w(5)=9$. This is not a natural weighting on $(E, \mathcal{F})$ because the ordering $(1,5,4,3,2)$ is not $\mathcal{F}$-feasible.

Now the greedy algorithm produces a solution $I_{\mathrm{GA}}=\{1,2,3\}$ with $w\left(I_{\mathrm{GA}}\right)=$ 13. But this is not an optimal solution of $P_{\max }(\mathcal{I}, w)$. The optimal solution is $I=\{3,4,5\}$ with $w(I)=19$. 
Example 6.8. Let $M=(E, \mathcal{F} ; \mathcal{B})$ be the (non-strict) cg-matroid given in Example 4.1. Then $\mathcal{I}=\mathcal{I}(M)=\{\emptyset,\{1\},\{2\},\{3\},\{4\},\{5\},\{1,2\},\{1,3\},\{2,3\},\{2,4\}$, $\{2,5\},\{3,4\},\{3,5\},\{4,5\},\{1,2,3\},\{2,4,5\},\{2,3,4\},\{2,3,5\}\}$.

Let $w: E \rightarrow \mathbb{R}_{\geq 0}$ be a weight function on $E$ defined by $w(1)=10, w(2)=1$, $w(3)=2, w(4)=8, w(5)=9$. This is a natural weighting on $(E, \mathcal{F})$ because there is an $\mathcal{F}$-feasible ordering $(1,5,4,3,2)$ which satisfies $w(1) \geq w(5) \geq w(4) \geq$ $w(3) \geq w(2)$.

Now the greedy algorithm produces a solution $I_{\mathrm{GA}}=\{1,3,2\}$ with $w\left(I_{\mathrm{GA}}\right)=$ 13. But this is not an optimal solution of $P_{\max }(\mathcal{I}, w)$. The optimal solution is $I=\{2,4,5\}$ with $w(I)=18$.

Note. A general model for matroids and the greedy algorithm is considered in [7] by U. Faigle and S. Fujishige; their model is a generalization of strict cg-matroids.

\section{Acknowledgments}

The author is grateful to the anonymous referee for his or her careful reading and suggestions to improve the presentation of the results. The author was supported by JSPS Research Fellowships for Young Scientists.

\section{References}

[1] K. Ando, Extreme point axioms for closure spaces, Discrete Math. 306 (2006), 3181-3188. Zbl 1110.54001 MR 2279056

[2] M. Barnabei, G. Nicoletti, and L. Pezzoli, The symmetric exchange property for poset matroids, Adv. Math. 102 (1993), 230-239. Zbl 0793.05035 MR 1252033

[3] M. Barnabei, G. Nicoletti, and L. Pezzoli, Matroids on partially ordered sets, Adv. Appl. Math. 21 (1998), 78-112. Zbl 0908.05025 MR 1623325

[4] F. D. J. Dunstan, A. W. Ingleton, and D. J. A. Welsh, Supermatroids, in Combinatorics (Oxford, 1972), Inst. Math. Appl., 1972, 72-122. MR 0340057

[5] P. H. Edelman and R. E. Jamison, The theory of convex geometries, Geom. Dedicata 19 (1985), 247-270. Zbl 0577.52001 MR 0815204

[6] J. Edmonds, Submodular functions, matroids, and certain polyhedra, in Combinatorial structures and their applications (Calgary, 1969), R. Guy et al. (eds.), Gordon and Breach, New York, 1970, 69-87; also in Combinatorial optimization-eureka, you shrink!, M. Jünger et al. (eds.), Lecture Notes in Comput. Sci. 2570, Springer, Berlin, 2003, 11-26. Zbl 1024.90054 MR 2163945

[7] U. Faigle and S. Fujishige, A general model for matroids and the greedy algorithm, Math. Program. 119 (2009), 353-369. Zbl 1180.90268 MR 2487009

[8] S. Fujishige, Submodular functions and optimization, 2nd ed., Ann. Discrete Math. 58, Elsevier, Amsterdam, 2005. Zbl 1119.90044 MR 2171629

[9] S. Fujishige, G. A. Koshevoy, and Y. Sano, Matroids on convex geometries (cg-matroids), Discrete Math. 307 (2007), 1936-1950. Z Zbl 1114.52018 MR 2320198 
[10] B. Korte, L. Lovász, and R. Schrader, Greedoids, Algorithms Combin. 4, Springer, Berlin, 1991. Zbl 0733.05023 MR 1183735

[11] J. Oxley, Matroid theory, Oxford Univ. Press, Oxford, 1992. Zbl 0784.05002 MR 1207587

[12] U. N. Peled and M. K. Srinivasan, Poset matching - a distributive analog of independent matching, Discrete Math. 114 (1993), 403-424. Zbl 0783.05037 MR 1217766

[13] Y. Sano, Rank functions of strict cg-matroids, Discrete Math. 308 (2008), 4734-4744. Zbl 1158.05020 MR 2438177

[14] A. Schrijver, Combinatorial Optimization. Polyhedra and Efficiency, Algorithms Combin. 24, Springer, Berlin, 2003. Zbl 1041.90001 MR 1956924(Vol. A), MR 1956925(Vol. B), MR 1956926(Vol. C)

[15] É. Tardos, An intersection theorem for supermatroids, J. Combin. Theory Ser. B 50 (1990), 150-159. Zbl 0727.05016 MR 1081219

[16] D. J. A. Welsh, Matroid theory, Academic Press, London, 1976. Zbl 0343.05002 MR 0427112

[17] H. Whitney, On the abstract properties of linear dependence, Amer. J. Math. 57 (1935), 509-533. JFM 61.0073.03 MR 1507091 